\title{
A Smart Segmentation Technique Towards Improved Infrequent Non-Speech Gestural Activity Recognition Model
}

\author{
Mohammad Arif Ul Alam ${ }^{1}$, Nirmalya Roy ${ }^{1}$, Aryya Gangopadhyay ${ }^{1}$, Elizabeth Galik ${ }^{2}$ \\ ${ }^{I}$ Department of Information Systems, University of Maryland Baltimore County \\ alam4@umbc.edu,nroy@umbc.edu,gangopad@umbc.edu \\ ${ }^{2}$ School of Nursing, University of Maryland Baltimore \\ galik@son.umaryland.edu
}

\begin{abstract}
Infrequent Non-Speech Gestural Activities (IGAs) such as coughing, diglutition and yawning help identify finegrained physiological symptoms and chronic psychological conditions which are not directly observable from traditional daily activities. We propose a new wearable smart earring which is capable of differentiating IGAs in daily environment with single integrated accelerometer sensor signal processing. Our prior framework, GeSmart, shows significant improvement in IGAs recognition based on smart earring which necessitates users to replace the earring batteries frequently due to its energy hungry requirement (high sampling frequency) towards fine-grained IGAs recognition. In this improved work, we propose a new segmentation technique along with GeSmart which takes the advantages of change-point detection algorithm to segment sensor data streams, feature extraction and classification thus any machine learning technique can perform significantly well in low sampling rate. We also implement a baseline traditional graphical model based gesture recognition techniques and compare their performances with our model in terms of accuracy, energy consumption and degradation of sampling rate scenarios. Experimental results based on real data traces demonstrate that our approach improves the performances significantly compared to previously proposed solutions. We also apply our segmentation technique on two benchmark datasets to prove the superiority of our technique in low sampling rate scenario.
\end{abstract}

Keywords: smart jewelry, behavioral health, change-point detection, energy efficiency, smart segmentation.

\section{Introduction}

Modeling and analyzing the physiological symptoms and psychological behaviors of older adults have a profound impact on future smart and connected older adults care. The fine-grained insights about the human health, wellness and independence obtained from the physiological and psychological data analysis if coupled with activities of daily living can help improve the mental health, stress disorders, ambulatory conditions, and social interactions of older adults. The wide availability of commodity smart healthcare appliances, stand-alone and integrated sensing devices make it increasingly easy to ubiquitously and continuously monitor an individual's health-related vital signals, activities, and behavior and to integrate such data into healthcare systems. We are witnessing early commercial activity, where a combination of body-worn medical and non-medical sensors (e.g., sensors to monitor blood oxygenation or accelerometers to monitor movement) and in situ sensors (e.g., thermal and motion detectors) continuously monitor and automatically determine an individual's context. Broadly speaking, context in smart health refers to a variety of dynamically changing states, related to either an individual's activities (e.g., ambulatory vs. sleeping), biomedical conditions (e.g., fatigue vs. anxiety), or behavioral conditions (e.g., shouting vs. agitation). In many health and wellness applications, such context enables critical capabilities, such as alerting a first responder if the individual is shouting for an abnormal period of time or flagging a health risk by analyzing wellness data related to continuous burping or hiccup after every day eating. In this paper, we particularly investigate the recognition and discovery of infrequent non-speech gestural activities (henceforth defined as IGAs) which are observable and detected; provide significant insights about 
the long-term well-being of the older adults. Our approach enables efficient abstraction and finer correlation of the activities of daily living with the acute physiological symptoms and chronic psychological conditions.

Providing both behavioral and physical health status in an unified setting is of utmost need for proactive healthcare. Mental disorders and cognitive impairments oftentimes evolve from chronic physiological symptoms and abnormal psychological behaviors. Suffering from different sort of mood disorders inhibit different patterns of infrequent gestures such as depression, sadness, crying, shouting etc. Likewise for different kinds of physiological health issues, the patient shows irregular gestures such as frequent coughing, burping, breathing problem etc. Therefore mental and physical health of older adults are correlated and if harnessed appropriately may provide meaningful microscopic physiological and psychological contexts. For example, a person feeling a headache from anxiety or anger might shout loudly or show irregular interpersonal traits. Thus the mental hygiene or physical wellness of a particular person can be inferred by monitoring the IGAs which reflect the emotional or behavioral state of the individual. On the other hand when a person shows infrequent gestures while being engaged in other activities, his or her body produces different kind of movements. The differences between these subtle movements, if captured and detected naturally could help infer the infrequent gestural activities.

Previous works have focused on human speech processing extracting features from acoustic signal to detect human voice and non-speech human sounds. While acoustic sensor can certainly help determine the sound gesture of human but undermine significantly the operational cost and life longevity of wearable devices due to its energy hungry operations. Acoustic signal recording, pre-processing, ambient noise reduction, features extraction and classification process cause huge computational overhead which rapidly drains out the battery power of source devices. Sound signals generating from other individuals, surrounding the target user may cause severe misclassification problem creating unavoidable false positive results. Moreover, continuous sensing of sound signals may cause serious privacy violations. On the other hand embedding sensors on myriad objects of daily living, such as microwaves and kitchen cabinets [4] or mounting them on the ceiling has challenging operational costs and battery-life issues. Individuals, particularly, older adults appear reluctant to continually wear multiple sensors on the body [2].

Activity recognition generally assumes that sensor data is indiscriminately represented as a time series, and that at any given moment in the time series the subject involves with single activity. Thus the time series is thought of as being partitioned into a number of non-overlapping intervals (windows), which are delimited by moments in time when the subject stopped performing one activity and started performing another. Previous work has treated activity detection as an online problem, and has rarely considered performance metrics other than accuracy. In this paper we consider accuracy as well as detection as performance metrics to provide more fine-grained granularity of our model prediction. We propose to use an energy efficient and computationally inexpensive accelerometer sensor in the form of a smart earring for detecting fine-grained gestural activities of the user. We are also interested in the feasibility of partitioning and classifying a time series data in real time. More specifically we aim to facilitate the following critical requirements:

- Long Battery Life: Earring has become very common for men and women to have both ears pierced, and it is becoming more acceptable for teenage and pre-teen boys to have both ears pierced as well. The advantage of smart earring based physical/gestural activity recognition is its ubiquitous usage of all time even during sleep. However, the most advantageous fact also creates the most disadvantageous charge. Since users keep it worn for long time, it is obvious that they are reluctant of changing batteries frequently. Moreover, if the batteries must be replaced often (every day or every week), not only will the primary benefit (freedom from wiring constraints and costs) of wireless networks be lost, but also many remote sensing applications may become impractical. Therefore, long battery life is essential in earring sensor networks.

- Small Form Factor: It is obvious that devices must be small enough to be embedded in their operating environment. This requirement affects the choice of batteries - even AA batteries are too bulky to power the sensor node, so using coin cell batteries is the only option in many situations.

- Low-sampling Rate: In our framework, sensor data is captured in the earring but transmitted, stored and processed by a remote node before it is transmitted to the central base station. The entire data streaming, transmission, storage and local processing are exhilarated with low sampling rate. However, low sampling rate costs the accuracy of the recognition process which also needs to be handled. 
Research Questions: Our investigations in this paper pursue the following research questions:

- Given the adaptation of activity recognition algorithms to help older adults in healthy independent living what sort of gestural activities may shed light on long-term physiological health and psychological behavior of older adults?

- What would be the best segmentation, feature extraction and classification techniques to detect such fine-grained gestural activities in very low sampling rate assuring maximum device battery power savings and computational cost reduction?

In this paper, we first use real life data traces from 20 subjects with a variety of different gestural activities (max 5). Then we explored a novel segmentation approach, change-point segmentation (CPS), based classification and compare with its performance over traditional Hidden Markov Model (HMM) based graphical approach, for segmenting and classifying realistic time series data for exploiting the emerging patterns in a variety of IGAs to detect and infer IGAs. In CPS approach, we use change-point detection to partition time series data into activity windows for classification. In HMM approach, we used the well-known technique of partitioning the time series into small fixed-length, nonoverlapping windows, predicting the activity type of each window using a base classifier, treating that prediction as the observable state of an HMM, and then finally solving the HMM for its hidden states. This approach begins with small windows of fixed-length, and uses an HMM to smooth windows together into larger activity intervals. We also applied feature selection method in both of our approaches. While each approach helps to successfully identify different gestural activities but fails to identify when the gestural events are either instantaneous or continuous in nature. Realizing this we proposed a novel change-point scoring based feature extraction and hybrid classification model for gestural activity recognition that exploits the abrupt changes in gestural signals along with its inherent pattern to obtain divergence estimation between the time-series samples. We validate the proposed approaches using real life data traces. Our work thus affirms how a microscopic gestural activity recognition model augmented with activities of daily living can provide practical insights that (a) helps capture the finer correlation of the activities of daily living with the acute physiological symptoms and psychological conditions, and (b) provide additional contexts which help devise novel interventions that can be effectively used in managing functional and cognitive health decline of older adults.

\section{Key Contributions:}

- Our key contribution lies in the proposed change point detection based gestural activity recognition approach (GeSmart) which represents the instantaneous perturbation of gestural signals as an abrupt change and continuous perturbation as a specific pattern and help detect the microscopic gestures. This provides a practical way to determine fine-grained discrimination of physiological and psychological health markers, without incurring the expensive and laborious in-situ laboratory testing.

- As a secondary contribution, we posit that low power, cheap accelerometer sensor is a potential option if integrated inside the smart jewelries (e.g., earrings, necklace etc) and provide better detection accuracy and substantial energy savings compared to the acoustic sensors.

- We also show that using change-point detection techniques for deciding when one activity ended within a time series and the next began, we can segment time-series data to achieve fine grained classification accuracy with endurable detection time even with low sampling rate.

- We evaluate the accuracies of our proposed two approaches using real life activity traces from 20 domestic users, collected over 15 minutes of session for each individual. Our results show that, given normal everyday patterns of domestic living, GeSmart can provide very high accuracy in identifying microscopic gestural activities $(\max \approx 96 \%)$, and significantly decrease the energy consumption $(\approx 30 \%)$. These results demonstrate the viability of the GeSmart approach, for both finer-grained gestural activity recognition and long-term healthy independent living. We also evaluate our smart segmentation technique on two benchmark datasets ([56], [57]) for activity recognition in terms of sampling rate and computational costs. 
The rest of the paper is organized as follows. We first discuss the related work and then present the high-level overview of the proposed GeSmart framework. We highlight our initial findings on gestural activity recognition based on our two different approaches. We then describe how change-point detection based gestural activity detection method can be integrated with our both of approaches. We develop an earring system using off-the-shelf commercially available accelerometer sensor and present our detailed experimental results. Finally, we identify future research directions and conclude our work.

\section{Related Works}

Most of the approaches in monitoring human gestures involve image or video feed analysis for tracking facial expressions or body postures. Early works for tracking gestures were unimodal which were based on only one criterion like vocal features, facial expressions, body postures or physiological changes $[18,19,14,15,13,12,11]$. Some researchers used microphones [45], weight detection panels [48], cameras [49], and water usage detectors [45] to detect gestural activities. Among the various wearable devices, the most popular wearable for activity detection purposes is the accelerometer. Besides being inexpensive, accelerometers tend to be small and lightweight, and so are fairly unobtrusive and user-friendly. Accelerometers also gather data at a high frequency, and as such may be used to collect a sizeable amount of data in a relatively short amount of time. After gathering data from different modalities, most of the work have focused on a supervised pattern classification algorithms to detect the gestures. But this approach fails to address the problem when a person has any overlapped gestures. The vocal features has also been used in gesture analysis based on the speech analysis techniques using signal processing. To differentiate between different variants of vocal sound Mel-Frequency Cepstral Coefficient (MFCC) has been applied and 66\% average accuracy for detecting 6 emotions has been reported [22]. [23] added acceleration of pitch and MFCCs to form feature streams. It has applied different machine learning techniques for stressed/neutral style classification and Gaussian SVM for 4class speaking style classification. Physiological signals like heart rate, skin conductivity, muscle activity etc have also been considered for inferring gestures achieving overall accuracy of $81 \%$ [15]. Recently researchers have proposed multimodal approaches where multiple sensor modalities have been considered simultaneously. [8][9] proposed the bimodal approach to capture human gestures by using both facial expression and body postures whereas [10] used multimodal approach where facial expressions, vocal features, body movements and gestures have been fused altogether. [24] proposed a rule based approach by applying classification of audio-visual data. The multimodal approaches indicate that the performance of gesture or emotion recognition can be improved by multi-modal sensor data fusion. A mobile sensing system leveraging the microphone sensor has been proposed to detect non-speech body sounds or gestural activities in [25].

Whether or not an accelerometer yields data that is discriminative for a set of activity types depends partially on where the accelerometer is worn on a subject's body. For example, an accelerometer worn on the ankle will be more discriminative for the activity of cycling than it would be if it was worn on the hip, and different types of arm movements will likely be discriminated only by an accelerometer worn on the arm. For this reason some researchers have considered to use multiple accelerometer systems to capture movement information from different parts of the body [50]. This approach can be cumbersome for the wearer, so a single accelerometer is preferred when it is reasonable to assume that it will be discriminative for the relevant set of activities. However, wireless sensor network based activity recognition imposes several other issues like performance expectancy in terms of hardware cost and processing delay, human training effort expectancy, social influence, facilitating condition and privacy issues $[69,70]$.

In our work, we propose to use an accelerometer sensor based earring to detect the subtle movements users made during the course of a gestural activity occurrence. Our work is closest to [16] which used wearable accelerometer sensor to identify social actions. The main difference between [16] and GeSmart is that former uses HMM model to analyze face-to-face interactive conversing behaviors (e.g., speaking, laughing, gesturing, drinking, or stepping) in a densely crowded social gatherings to find out the correlation between movement acceleration and a person's social activity, ability of talking in a group with known/unknown persons or power of dominating in a group conversation. While [16] focused on building a model for only conversing behaviors using wearable accelerometer sensor incorporated with a badge (which is not ubiquitous), we focus in this paper to identify infrequent gestural activities which are independent of any specific posture (i.e., standing, sitting, running etc.) or predefined environment (i.e., alone or 


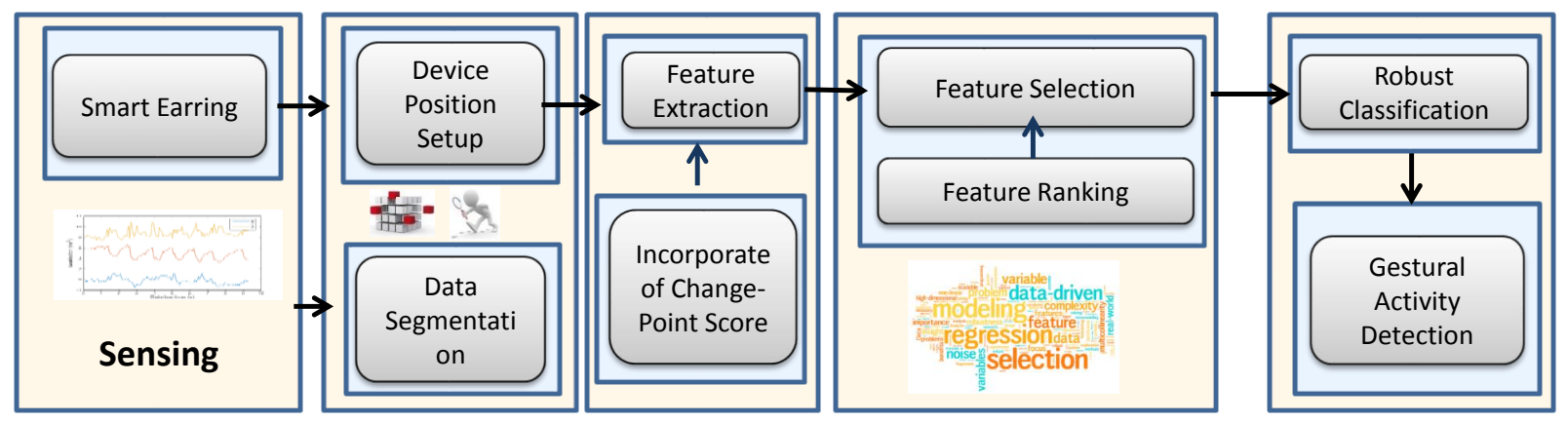

Figure 1: An overview of our model

crowded) using ubiquitous device (i.e., earring). In general it is easier to detect fine-grained movements when the user posture and context are known a-priori such as conversing in a standing position [16]. But in this paper we focus on building a generic gestural activity recognition model independent of any specific location or postural position of a user. Indeed we particularly focus on specific gestural activities rather than social interactions which have long-term correlation with the physiological and psychological health of a person. In the past few years researchers have begun to recognize the need to test on realistic free-living data $[55,58]$. The time required to detect that a change in activity has occurred was considered in [59], but in the context of the very different problem of video activity recognition. We consider the feasibility of performing accelerometer based IGA recognition in real time by using both accuracy and detection time as performance metrics.

Reducing sampling rates to save energy is a well studied research topic. Some works such as [44] point out that to achieve high classification accuracy, it is not necessary for sensors to work at full sampling rate. The paper [46] statically addresses the tradeoff between accuracy and energy consumption. For both time and frequency domain features, it reports that in the sampling rate dimension an accuracy knee exists. Below the knee, there is a significant accuracy degradation. It suggests using the sampling rate at the knee to save energy while enjoying a relative high accuracy. In addition, Chu [47] tries to achieve an optimal energy-latency-accuracy tradeoff for mobile sensor data classification. However, we proposed a novel approach of segmentation by accumulating change-point scoring based feature extraction method that significantly enhance IGAs recognition performances in lower sampling rate which confirms energy savings as well as computational cost.

\section{An Overview of GeSmart Framework}

Fig. 1 shows a schematic representation of our proposed GeSmart model. It consists of the following logical steps. 1) Data Collection: gathering the accelerometer readings from Chronos through the bluetooth access point. 2) Device Position setup: Calculating average change-point score for each gestural activities from four different body positions. 3) Data Segmentation: Derive data segments corresponding to movement components (i.e., framing, windowing etc.); 4) Feature Extraction: Estimate accelerometer data features from each data segment incorporating change-point score; 5) Feature Selection: Rank features according to the contributions of achieving separation among classes associated with different change-point scores; and select feature sets that minimize the overlap among classes as associated with different change-point scores; 6) Robust Classification: Use 10-fold cross validation to estimate the quality of change-point score based classification for individual IGAs; finally, 7) Gestural Activity Detection: Apply the hybrid classification model on real data traces to detect the fine-grained IGAs. We follow two distinguished approach in this regard. Fig 2 illustrates the detail of our approach. 


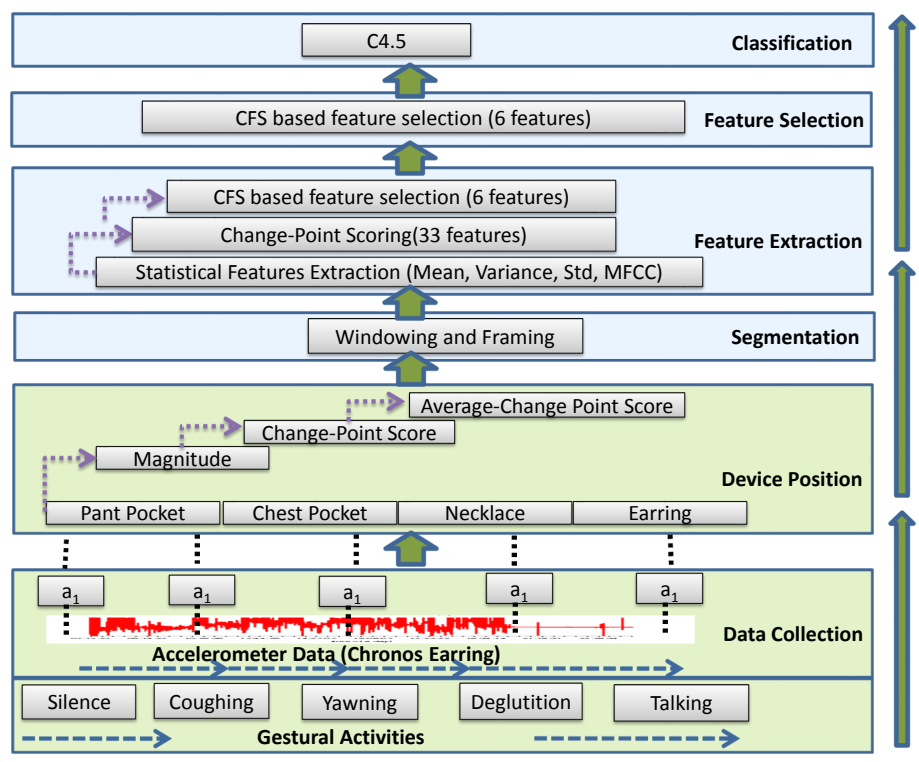

Figure 2: Flowchart of proposed model

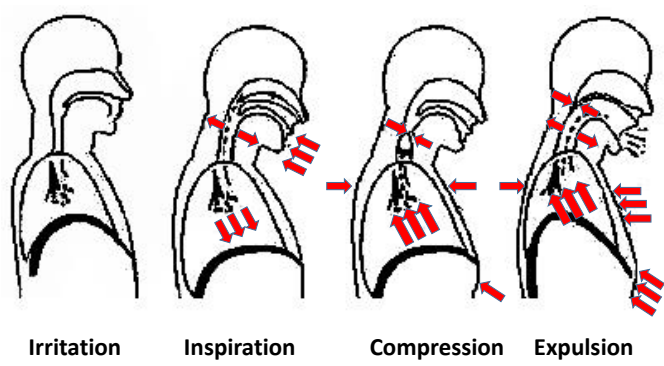

Figure 3: Normal Coughing Consists of four events

\section{Gestural Activity Detection: Initial study and Findings}

Given our focus on detecting gestural activities, we first present the challenges of capturing and recognizing gestural activity's acceleration patterns in perspective of human body movement and motion. The goal here is to establish that with only an accelerometer based system we can leverage the non-speech body sound associated with a variety of IGAs.

\subsection{Selection of Gestural Activities}

The major goal of this study is to provide both behavioral and physical health status in terms of non-speech gestures. Towards this broader perspective, we choose 5 important non-speech gestural activities which are closely relevant to proactive healthcare and abnormal physical behaviors: (i) silence, (ii) talking, (iii) coughing, (iv) yawning and (v) deglutition. 'Silence' is the most common form of human non-speech gesture which defines a state when the person does no significant sound through her mouth and stays the mouth close to normal position. The silence movement does not change the jaw and ear positions of the face. However, it also can be considered as a separator from one IGAs to another due to its universal presence in-between all IGAs natural performance. 'Talking' is the speaking state of human gesture which has been considered in this study to distinguish it from other IGAs. 'Coughing' is a sudden and often repetitively occurring reflex which helps to clear the large breathing passages from secretions, 


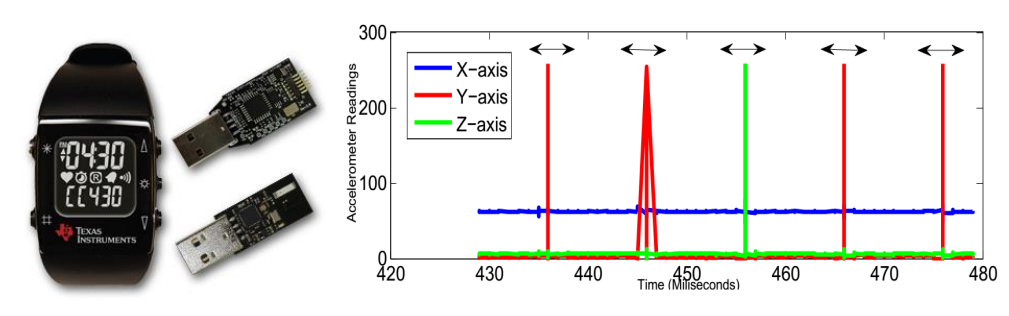

Figure 4: (1) Chronos. Wrist Watch, CC1111 USB RF access point, eZ430 USB programming and debugging interface (b) Coughing data from Chronos used as earring

irritants, foreign particles and microbes. 'Deglutition' or 'Swallowing' is the process in the human or animal body that makes something pass from the mouth, to the pharynx, and into the esophagus, while shutting the epiglottis. Swallowing is an important part of eating and drinking. If the process fails and the material (such as food, drink, or medicine) goes through the trachea, then choking or pulmonary aspiration can occur. In the human body the automatic temporary closing of the epiglottis is controlled by the swallowing reflex. 'Yawning' is a reflex consisting of the simultaneous inhalation of air and the stretching of the eardrums, followed by an exhalation of breath. 'Yawning' most often occurs in adults immediately before and after sleep, during tedious activities and as a result of its contagious quality. It is commonly associated with tiredness, stress, sleepiness, or even boredom and hunger, though studies show it may be linked to the cooling of the brain [42]. The above activities can be related to each other based on their nature. For example, 'Coughing' can be followed by 'Deglutition', 'Silence' or 'Talking' which represent different form of pattern and may consist of clinical significance in nature of 'coughing' detection. However, 'Coughing' followed by 'Yawning' could be an indication of sleep disturbance caused by couching. However, our baseline framework has opportunity to add more activities too.

\subsection{Anatomy of Gestural Patterns}

The instantaneous or continuous periodicity of gestural events and their impact on human motion pose significant challenge on detecting them successfully. Different gestural activities has different intensity and motion characteristics, which may posit diagnostically valuable movement information to distinguish them. These underpinning characteristics are correlated with physical constraints of a person generating unique capturable human motion patterns at occurrences. Every spontaneous gestural activity (e.g., coughing, yawning etc.) occurs when a sequence of events is stimulated by the presence of sputum or foreign particles in the main, central airways of a person [29]. For example, in case of normal coughing the sequence of events are referred to as the sequence of irritation, inspiration, compression and expulsion [29]. Irritation is an abnormal stimulus (inflammatory, mechanical, chemical or thermal) which provokes sensory fibers to send impulses to the brain's medullary cough center. In the inspiration phase the glottis becomes wide open due to reflex muscle contraction. Movement of glottis, respiratory muscles and the branches of the bronchus are closely tied during the course of cough phase. Thus these four phases describe the major effects of the cough reflex. Each phases causes unique pattern of movement associated with the human body as shown in Fig. 3. In Fig. 3, we see a sudden downward acceleration change due to the movement of head from irritation to inspiration state. Then inspiration to compression state causes almost no acceleration downward of head. Again, we see sudden upward acceleration change of head from compression to expulsion state. To capture these slightest movements and acceleration changes of the user, we place an accelerometer based system, Chronos (Fig. 4) corresponding to different body position as a smart jewelry such as earring and necklace.

\subsection{Recognizing Gestural Events}

Although we are able to define different micro events that construct human gestures, those micro events' duration, occurrence sequence and acceleration features (i.e., $\mathrm{x}, \mathrm{y}$ and $\mathrm{z}$ axis data features) in terms of body movements vary from one gesture to another. For example, normal cough consists of four events: irritation, inspiration, compression and 

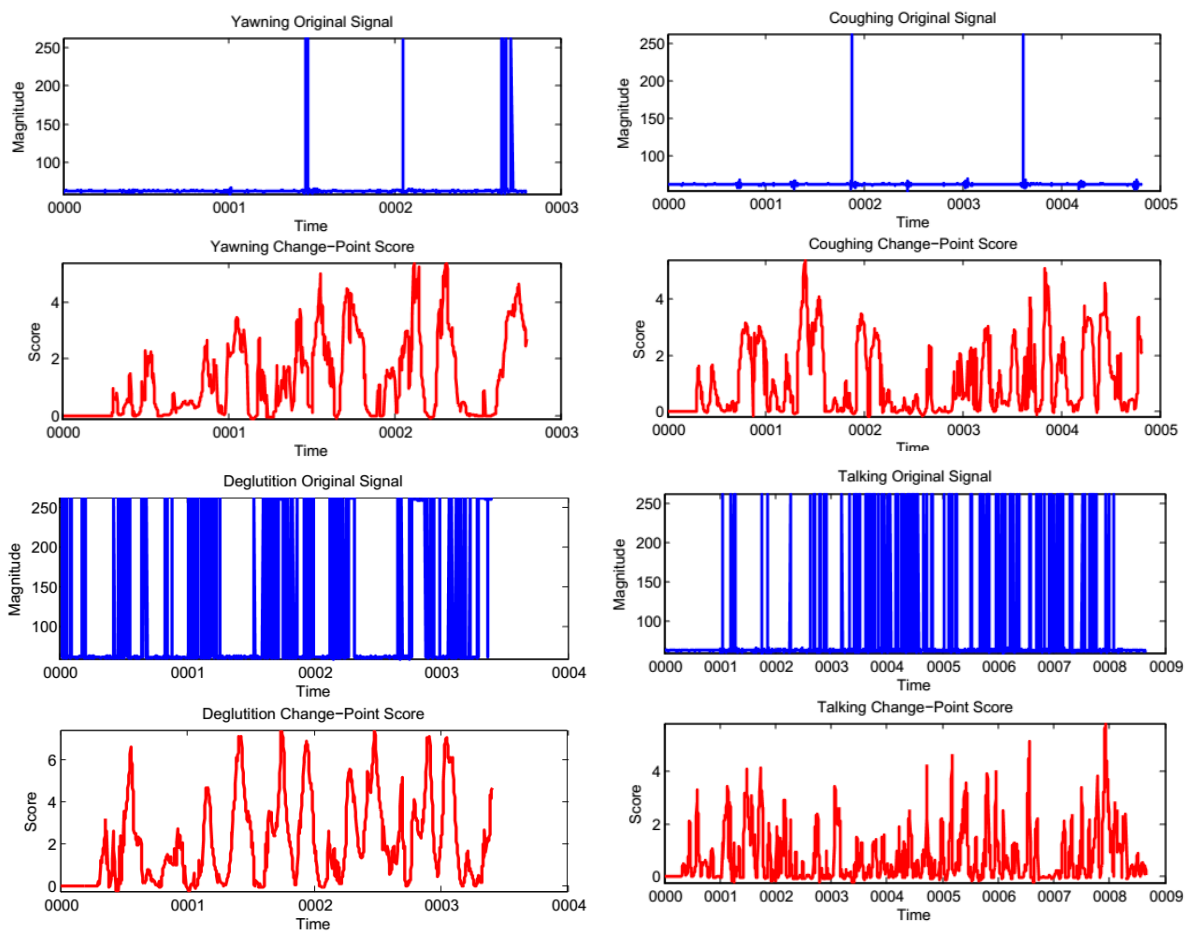

Figure 5: Acceleration (magnitude $=\sqrt{x^{2}+y^{2}+z^{2}}$ ) and corresponding Change-Point Score of talking, coughing, yawning and deglutition

expulsion but normal yawning consists of only irritation, inspiration and expulsion with different duration ratio [29]. Even in case of dry coughing caused by tuberculosis, consecutive coughing may cause intense pain in the throat creating several extra compression events. Thus it is challenging to recognize gestural activities using an unified model. While using Chronos as an earring to capture these coughing events' acceleration due to its impact on head, we note that the $\mathrm{x}$ and $\mathrm{z}$ axis accelerometer sensor data are always steady, but $\mathrm{y}$ axis acceleration increases when the transition from irritation to inspiration occurs and decreases when the transition from compression to expulsion occurs (see Fig. 4). Fig. 5 depicts that different gestural activities has almost similar movement (i.e., acceleration) patterns making the classification problem more challenging. To distinguish these similar statistical features we propose to use change-point scoring method on each statistical feature which helps capture the fine-grained changes between the gestural activities. Fig. 5 shows the change-point scores of magnitudes applied on each statistical feature which enlightens the unique pattern for each of the gestural activity measures. Next we focus on developing the smart earring prototype, finding out its most informative position on the body, and designing robust classification and change point detection based hybrid classification model.

\subsection{Device Setup and Customization}

The goal for selecting a device for our earring prototype development was mainly cost, form factor, rapid customization and ease of deployment and data collection. The Texas Instruments Chronos development [38] was found to fit our needs and used for the development. The Chronos is a development platform built around an MSP430compatible system-on-chip with an integrated wireless modem. Communications between a computer and the Chronos modem was done by a USB-interfaced "access poin" that comes with the kit. Data between the host system and the access point is communicated through a virtual COM port abstracted by the access point driver distributed by Texas Instruments. The accelerometer included with the Chronos platform is a Bosch BMA250 [40]. The BMA250 exposes an SPI and I2C interface for communication, and internally utilizes a 10-bit analog-to-digital converter. Serial communications are the limiting factor in sampling rates, offering a bandwidth of up to $1000 \mathrm{~Hz}$. The BMA250 has 

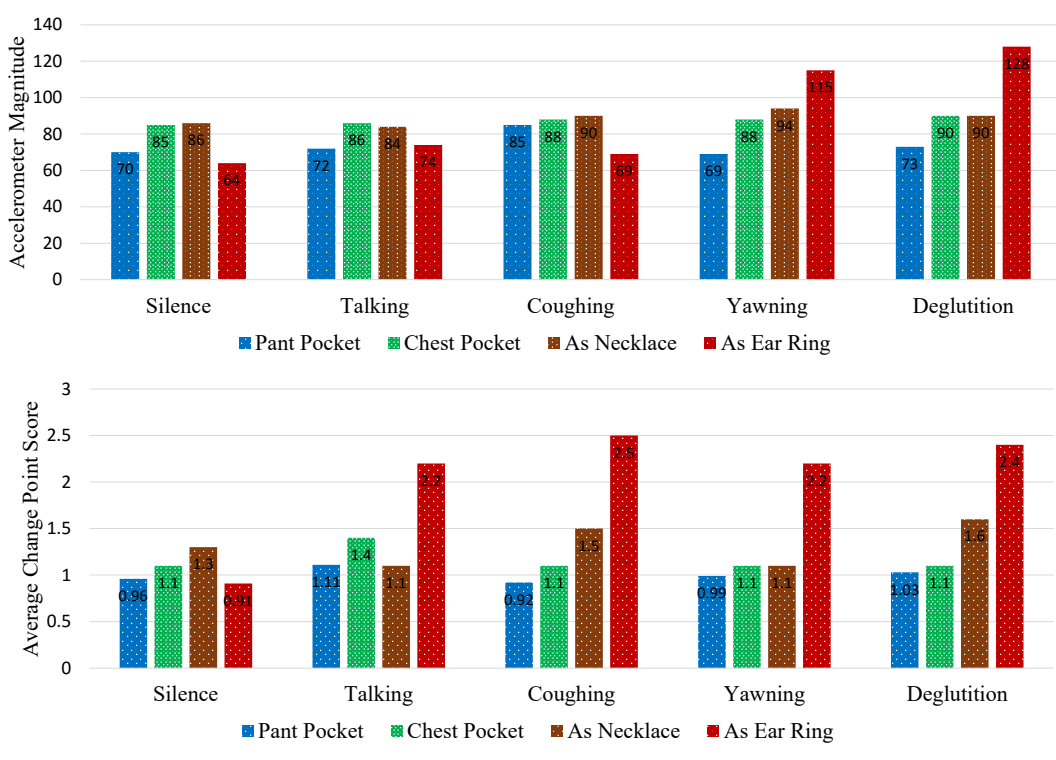

Figure 6: Comparing different body positions (pant pocket, chest pocket, neck, ear) to capture different types of gestural motion

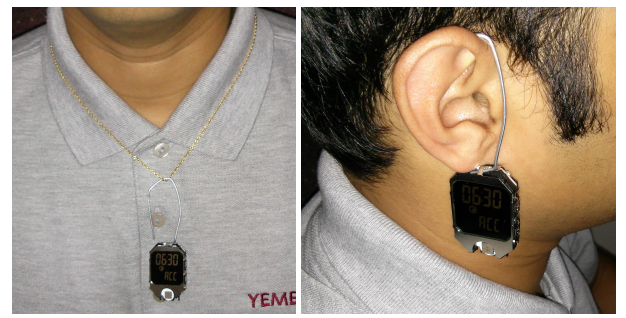

Figure 7: Chronos in different position

a programmable range of $2 \mathrm{~g}$ to $16 \mathrm{~g}$. The Chronos ships pre-programmed device with an evaluation firmware that demonstrates the features of the device, including the reading of raw accelerometer data, but the software from Texas Instruments does not provide an option to save the data received to disk. The simple binary serial protocol used is not described in Texas Instruments documents, and collecting information about the protocol by reading the publicly available source code for the firmware was found to be impractical. In an effort to develop interfacing software, communications between the Texas Instruments host-side software and the virtual COM port were monitored, and the protocol was elucidated by analyzing those communications. The protocol was re-implemented, and software was developed in $\mathrm{C \#}$ to attach to the virtual COM port provided by the Chronos access point and poll for accelerometer data. Data was saved to disk in a CSV format.

\subsection{Device Position Setup}

We conducted various experiments to find out the most informative device position to capture the most significant acceleration of human body movements for different gestural level activities. We performed extensive study on real data traces collected from 10 users using our prototype system to confirm the most informative position on the body. This test consisted of two parameters: the first being body position (pant pocket, chest pocket, neck and ear) and the second being gestural activities (silence, talking, coughing, yawning and deglutition). We recorded the five types of gestural activities' accelerometer data from the device and took the average accelerometer magnitudes and average change point scores of accelerometer magnitude (magnitude $=\sqrt{x^{2}+y^{2}+z^{2}}$ ) to compute the average acceleration 
changes in each gestural activity. Fig. 6 presents the change point score with respect to different body positions and gestural activities. The change-point score we used is an abrupt signal changes measure based on subsequence pattern matching [30]. We describe details and significant impact of this algorithm on gestural activity recognition in later part of our paper. We compared the average change-point scores and average magnitudes of the captured accelerometer data with respect to different body positions for different gestures. Fig. 6 shows that considering average magnitudes, it is impossible to detect the best position on the body for gestural activity recognition. But considering average change-point scores, it is noted that among the four locations, the ear gives us greater average change-point scores for all types of gestural activity, except silence. Intuitively, the breathing motion only affects chest and neck creating abrupt changes in acceleration than at pant pocket or ear. This continuous changes in acceleration due to the inherent breathing activity poses challenges to detect our finer IGAs. In fact, through our device position experimentation as shown in Fig. 6 in presence of a variety of IGAs along with the continuum regular ADLs, we establish that the position ear is always less affected by any external noise sources. To reduce this breathing noise, prior researchers $[16,20,21,5,6,7,3]$ proposed to use multiple levels of noise reduction methods. In our case, intelligent determination of this position a-priori help reduce the unwanted noise created from the breathing gestural activity. Fig. 6 showed that the position ear is more informative and noise free than other three positions; pant pocket, chest pocket or necklace. Therefore, we postulate that given our goal of capturing a wide range of gestural activity events in presence of normal ADLs, the position ear is the most informative and noise free location for the GeSmart jewelry device design and real deployment.

\section{Change Point Detection Algorithm}

Change point detection refers to identify the instances when the probability distribution of a stochastic process or time series changes. As we note previously that the standard statistical features fail to exploit the abrupt changes in gestural signals we propose to use change-point detection to capture the signal divergence. We design a hybrid classification model and use the relative Pearson divergence as a divergence measure estimated by a method of direct density-ratio estimation method [30]. We first mathematically describe the evolution of change point scoring for one dimensional time series sample of single valued sensor, and then consider multi-dimensional abrupt changepoint detection estimation associated with the combination of three axis-accelerometer observational values and their standard statistical features. Finally we describe how we can apply change-point detection algorithm in time series data stream segmentation.

\subsection{Change-point Scoring Method}

To explain the change-point scoring method, consider $y(t)$ as a 1-dimensional time series with single subsequence sample at time $\mathrm{t}$ where $y(t)=[\mathrm{x}$-axis reading, $\mathrm{t}]$. Then, the subsequence of time series at time $\mathrm{t}$ with length $\mathrm{k}$ be,

$$
Y(t)=\left[y(t)^{T}, y(t+1)^{T}, \ldots, y(t+k-1)^{T}\right]^{T} \in \mathbf{R}^{\mathbf{k}}
$$

where $y(t)^{T}$ represents the transpose of $y(t)$. Now, consider $\mathbf{Y}(\mathbf{t})$ be a set of $n$ retrospective subsequence samples starting at time t. Then,

$$
\mathbf{Y}(\mathbf{t})=Y(t), Y(t+1), \ldots, Y(t+n-1) \in \mathbf{R}^{k \times n}
$$

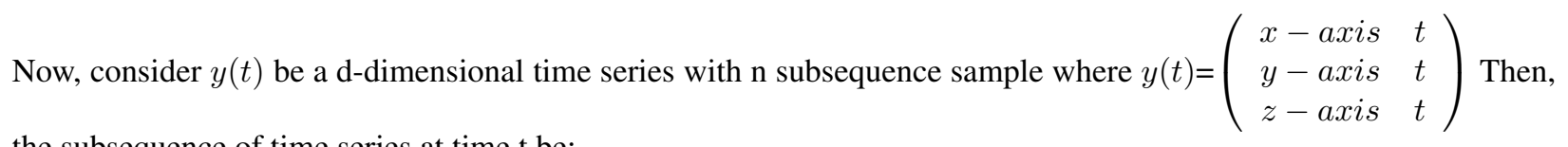
the subsequence of time series at time $t$ be:

$$
\mathbf{Y}(\mathbf{t})=Y(t), Y(t+1), \ldots, Y(t+n-1) \in \mathbf{R}^{d k \times n}
$$

$\mathbf{Y}(\mathbf{t})$ forms a Hankel matrix and plays a key role in change-point detection based on subspace learning [34]. In our model, we considered $k=10, n=50$ and $d=3$. We compute the dissimilarity measure between two consecutive segments $\mathbf{Y}(\mathbf{t})$ and $\mathbf{Y}(\mathbf{t}+\mathbf{n})$, and use it as the plausibility of change points i.e., the higher the dissimilarity measure 


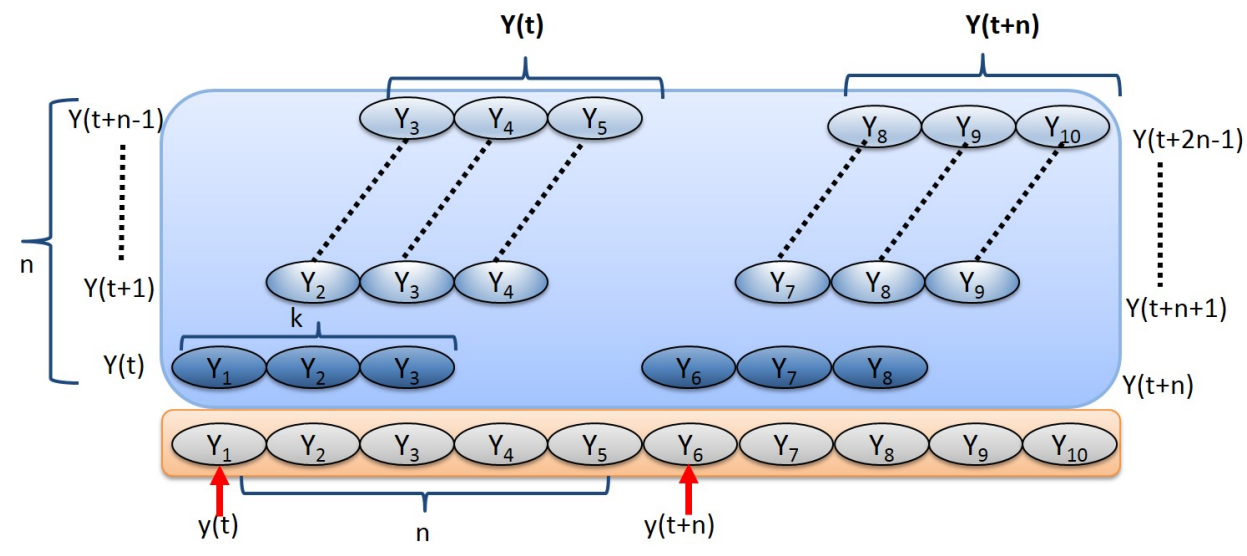

Figure 8: One-dimensional time-series data.

is, the more likely the point is a change point as depicted in Fig 8. Mathematically, we represent the dissimilarity measure as follows,

$$
D\left(P_{t} \mid P_{t+n}\right)+D\left(P_{t+n} \mid P_{t}\right)
$$

where $P_{t}$ and $P_{t+n}$ are probability distributions of samples in $\mathbf{Y}(\mathbf{t})$ and $\mathbf{Y}(\mathbf{t}+\mathbf{n})$, respectively. $D\left(P \mid P^{\prime}\right)$ denotes the $f$-divergence [35]. We use Pearson Divergence Estimation(PE) [36] which is a modified version of $f$-divergence and represent PE divergence as follows,

$$
P E\left(P \mid P^{\prime}\right)=\frac{1}{2} \int p^{\prime}(Y)\left(\frac{p(Y)}{p^{\prime}(Y)}-1\right)^{2} \times d Y .
$$

Where $p(Y)$ and $p^{\prime}(Y)$ are probability densities. Since the probability densities $p(Y)$ and $p^{\prime}(Y)$ are unknown in practice, we cannot directly compute the $f$-divergence and thus the dissimilarity measure. One simple solution of this problem is to perform density estimation and plug the estimated densities $p(Y)$ and $p^{\prime}(Y)$ in the definition of the $f$-divergence. In this paper, we follow [66] proposed direct density-ratio estimator method. The basic idea of this method is to learn the density-ratio function $\frac{p(Y)}{p^{\prime}(Y)}$ without going through separate density estimation of $p(Y)$ and $p^{\prime}(Y)$. An intuitive rationale of direct density-ratio estimation is that knowing the two densities $p(Y)$ and $p^{\prime}(Y)$ means knowing their ratio, but not vice versa; $p^{\prime}(Y)$ the ratio $p(Y)$ and $p^{\prime}(Y)$ does not necessarily mean knowing the two densities $p(Y)$ and $p^{\prime}(Y)$ because such decomposition is not unique. This implies that estimating the density ratio is substantially easier than estimating the densities, and thus directly estimating the density ratio would be more promising [67].

To estimate PE divergence, we use relative density-ratio estimator (RuLSIF) [37]. Considering $\alpha$-relative PEdivergence for $0<\alpha<1$, we have,

$$
P E_{\alpha}\left(P \mid P^{\prime}\right)=\int p_{\alpha}^{\prime}(Y)\left(\frac{p(Y)}{p^{\prime}(Y)}-1\right)^{2} \times d Y .
$$

where $p_{\alpha}^{\prime}(Y)=\alpha p(Y)+(1-\alpha) p^{\prime}(Y)$ is $\alpha$-mixture density. So the final dissimilarity measure is

$$
S(i)=P E_{\alpha}\left(P \mid P^{\prime}\right)+P E_{\alpha}\left(P^{\prime} \mid P\right)
$$

where $S(i)$ represents the change-point score. Given $\alpha$-relativedensity - ratio estimator $\hat{g}(\mathbf{Y})$, an approximation of the PE divergence is constructed as:

$$
P \hat{E}_{\alpha}=-\frac{\alpha}{2 n} \sum_{i=1}^{n} \hat{g}\left(\mathbf{Y}_{\mathbf{i}}\right)^{2}-
$$




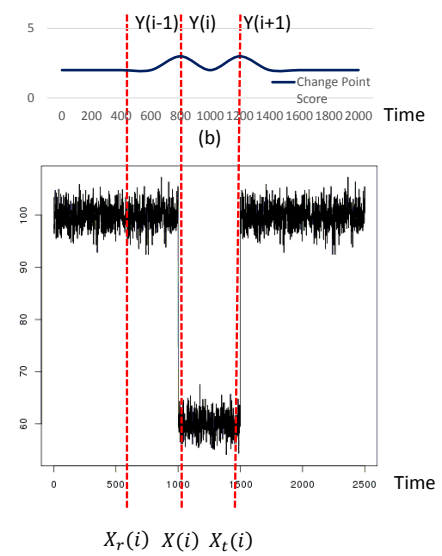

(a)

Figure 9: Change point detection based segmentation

$$
\frac{1-\alpha}{2 n} \sum_{j=1}^{n} \hat{g}\left(\mathbf{Y}_{\mathbf{j}}^{\prime}\right)^{\mathbf{2}}+\frac{\mathbf{1}}{\mathbf{n}} \sum_{\mathbf{i}=\mathbf{1}}^{\mathbf{n}} \hat{\mathbf{g}}\left(\mathbf{Y}_{\mathbf{i}}\right)-\frac{\mathbf{1}}{\mathbf{2}}
$$

We include the estimated change-point score $S(i)$ of the most popular statistical features in our model. We use the change-point algorithm implementation [39] to estimate the change-point score of a variety of gestural activities as shown in Fig 5.

\subsection{Change Point Detection Based Segmentation}

For this approach, we split data stream (x, y and $\mathrm{z}$ axis of accelerometer data) into non-overlapping segments using statistical field of change-point detection. Change-point detection in general is used to analyze time series data from dynamic systems, including failure detection [60], quick detection of attacks on computer networks [61], monitoring of heartbeat fluctuations during sleep [62]. Very few of prior works applied change-point detection in segmentation but most of these were related to vocal or non vocal sound segmentation[65, 64]. We are the first of a kind to apply change-point detection in segmenting accelerometer data stream. We identify the best change-point score threshold to segment time series data, apply supervised classification techniques and show how we can get more fine-grained performance in classifying infrequent gestural activities. To explain how Change-point scores is used for segmentation, we have to consider each tick of a time series as some sort of probability distribution, but that the distribution may suddenly change as time passes. The goal of change-point detection is to predict when these changes have occurred, a significant score is generated for each time tick (described in previous section). If the score is above a given threshold a change is predicted to have occurred between that tick and its immediate predecessor. This change point is considered as a partition between two consecutive segments. We then extract the features of each segment throughout the data stream for next level of classification. Fig 9(a) illustrates the data stream where $X_{r}(i)$ and $X_{t}(i)$ represent two consecutive sub-sequence of data stream associated with the $i^{\text {th }}$ tick. Fig 9(b) represents the changepoint score based segmentation. Here, change-point score $S(i)$ goes below the minimum threshold resulting an end of previous segment $Y(i-1)$ as well as a start of next segment $Y(i)$.

\section{Hidden Markov Model}

A Hidden Markov Model (HMM) contains a set of hidden states $X=\left\{X_{0}, X_{1}, \ldots, X_{w}\right\}$ and a set of observed states $O=\left\{O_{1}, O_{2}, \ldots, O_{w}\right\}$ (Fig 10) in its structural definition. There exists two indices $i$ and $j$ where $i<j, i$ is happened before $j$, each hidden state in the model contains a value from the state space $U=\left\{U_{1}, U_{2}, \ldots, U_{\ell}\right\}$, and each observed state contains a value from the observation space $V=\left\{V_{1}, V_{2}, \ldots, V_{m}\right\}$. Then the value of an 


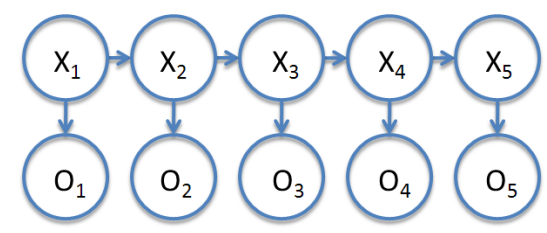

Figure 10: Hidden Markov Model Representation

observable state $O_{i}$ is dependent only the value of the corresponding hidden state $X_{i}$, and that the value of any hidden state $X_{i}$ is dependent only on the value of its immediate predecessor $X_{i-1}$. The dependencies between hidden states and their followers are assumed to be described by a stationary stochastic process known as the transition model, $T: U^{2} \rightarrow[0,1]$. The dependencies between a hidden state and its adjacent observable state are assumed to be part of a separate but also stationary stochastic process known as the observation model, $S: U \times V \rightarrow[0,1]$. The usual approach to estimate $T$ and $S$ given only $O$ is an application of Expectation Maximization (EA) known as the BaumWelch algorithm [63]. However, let a training $H M M\left\langle X_{t r}, O_{t r}\right\rangle$ with the same model parameters is given, and the values of all of its hidden states as well as its observable states are known. Then, $T$ and $S$ are approximated by a global maximum likelihood estimators. Finally, if all of the values of $O$ are known, and we are given a $\hat{T}$ and an $\hat{S}$ estimated from a training HMM, then the goal we are interested in is to use that information to find the most likely values for each state in $X$. We used Viterbi algorithm to determine the most likely values [52].

\section{Methodology}

We propose two hybrid classification techniques based on change point detection method combining traditional feature based technique with additional change point score based filtering. Detecting abrupt changes in time-series data, relying on change-point detection methods, can be classified into two categories:

- Real-time detection, targets applications that require immediate responses such as robot control, intrusion detection etc..

- Retrospective detection, useful for more robust and abrupt signal change detection although detection may require longer reaction periods.

In this paper, we propose to incorporate retrospective change point detection based method along with two different traditional segmentation and classification technique to capture the finer movement changes in IGAs.

\subsection{Data Collection}

We recruited 20 student volunteers (5 female and 15 male) with different heights (mean 170.3 centimeters with standard deviation 5.83 centimeters), weights (mean 176.3 pounds with standard deviation 15.3) and ages (19 to 30 years old with mean 25.3 and standard deviation 2.6) to collect five different gestural activities (i.e., silence, talking, coughing, yawning and deglutition) in two postural positions (i.e., standing and sitting). The accelerometer sampling rate is 22 samples/second. The participants were asked to wear the "Chronos" on their ear and to adjust the position of the hook behind their ear such a way that it seems like he or she is wearing an earring. They were asked to perform 5 different gestural activities in two different postural states. The types of gestural activities and a short description of each task are listed in Table 1. Most of the previous works considered talking and silence activities as noise in their classification methods [25][16]. For example, [25] focuses on non-body sounds where talking and silence created some noises in their classification methods creating the need of filtering them out. In our system, the choice of device position (i.e., use of device as earring) and change-point detection algorithm conform fine grained classification for both silence and talking. In total, each of our participants contributed at least 15 minutes of continuous recordings consisting of a controlled sequence of five gestural activities. Table 1 shows the detail description of our captured gestural activities. 
Table 1: List of gestural activities with distribution

\begin{tabular}{|c|c|c|c|c|}
\hline Index & Gesture & Description & \# Samples & Time Distribution (Minutes) \\
\hline 1 & Silence & Without any gestural activities & & 181.6 \\
\hline 2 & Coughing & $\begin{array}{c}\text { Natural two coughing } \\
\text { (single, consecutive two \& three) }\end{array}$ & $\begin{array}{c}263 \\
(186,49 \& 28)\end{array}$ & $\begin{array}{c}12.6 \\
(7.7,3.2 \& 1.7 \text { respectively) }\end{array}$ \\
\hline 3 & Yawning & Yawning as natural as possible & 285 & 19.3 \\
\hline 4 & Deglutition & Natural water deglutition & 281 & 31.15 \\
\hline 5 & Talking & Normal talking & 220 & 55.3 \\
\hline & & Total & & 300 \\
\hline
\end{tabular}

\subsubsection{Description of Data Collection Session}

We build a video manual instructing how the participants require to perform the IGAs. The manual starts with an instruction about the entire experiment, the goals of the experiment, final outcomes and a consent form. Then, the participants are asked to read several segments of paragraphs in natural talking voice chosen from recent articles published in New York Times newspaper displayed on the screen. Then, the video displays an instruction requesting to cough for 1-3 consecutive times as natural as possible. Similarly the participants are asked through the video instruction to perform all of the IGAs and silence activities in two different positions, (i) sitting and (ii) standing. A glass of water is kept in-front of the participants to help perform 'deglutition'. However, the participants are allowed to do any of the 5 activities at any time in the middle of the session. A webcam camera enabled with audio recording has been configured with time to record entire session. The nature of 'coughing' is designed very carefully in three coughing forms (single, two consecutive and three consecutive) as rest of the activities (i.e., 'silence', 'talking', 'yawning' and 'deglutition') do not have diversity like 'coughing' in natural scenario.

Two trained graduate student annotators are recruited to properly annotate the ground truth of the entire session and another one graduate student are recruited to validate the annotation. To help them annotate the ground truth, a continuous graph of acceleration magnitude (similar to Fig 5 spectogram) has been displayed along with the video. Table 1 shows the details of our dataset, class distribution and sub-class of coughing (single, consecutive two and consecutive three coughing samples) distribution with sample sizes.

To examine the acceleration characteristics of the collected accelerometer data in different gestural activities, we plot their corresponding spectrograms in Fig. 5. Spectrogram illustrates a visual representation of the $\mathrm{x}, \mathrm{y}$ and $\mathrm{z}$ axis spectrum of a gestural event as it varies with time. Silence is not shown separately because it is always present in between two consecutive gestural events. The distinct spectral pattern is not clearly visible in the original graph of $\mathrm{x}$, $\mathrm{y}$ and $\mathrm{z}$ axis spectrum, while change-point scoring for all of the gestural activities generates a distinct spectral pattern.

\subsection{Feature Extraction}

At first, we split each time series data stream into a set of non-overlapping windows of feature vector through a trial and error method. We used 18 statistical features that have uni-axial and bi-axial statistical properties. The uni-axial statistics were applied to data from each axis separately and the bi-axial statistic was applied to data from each of the $C_{2}^{3}=3$ possible pairs of axes for a total of $18 * 3+3=57$ features [54]. We briefly describe our feature sets bellow.

The sum and the sample mean act such properties that as more intense activities will tend to involve higher rates of acceleration during movement. We also used the $10^{t h}, 25^{t h}, 50^{t h}, 75^{t h}$, and $90^{t h}$ percentiles of the data, as well as signal power and log energy as supplemental measures of overall activity intensity. The standard deviation, coefficient of variation, amplitude, zero crossings count and the interquartile range are useful for discriminating between activities with a consistent level of intensity and activities. Lag-one-autocorrelation, skewness, kurtosis, and peak intensity are useful for discriminating between activities that tend to be similar in their overall intensity and variation in intensity. The correlation coefficient feature, which discriminates between activities where acceleration values in one axis are predictive of acceleration values in another axis, versus activities where that is not the case. Table 2 shows the details 
Sum of values of a period of time: $\sum_{i=1}^{T} x(i)$.

Mean: $\mu_{x}=\frac{1}{T} \sum_{i=1}^{T} x(i)$.

Standard deviation: $\sigma_{x}=\sqrt{\frac{1}{T} \sum_{i=1}^{T}\left[x(i)-\mu_{x}\right]^{2}}$.

Coefficients of variation: $\frac{\sigma_{x}}{\mu_{x}}$.

Peak-to-peak amplitude: $\max \{x(1), \ldots, x(T)\}-\min \{x(1), . ., x(T)\}$.

Percentiles: $10^{t h}, 25^{t h}, 50^{t h}, 75^{t h}, 90^{t h}$.

Interquartile range: difference between the $75^{\text {th }}$ and $25^{\text {th }}$ percentiles.

Lag-one-autocorrelation: $\frac{\sum_{i=1}^{T-1}\left[x(i)-\mu_{x}\right]\left[x(i+1)-\mu_{x}\right]}{\sum_{i=1}^{T}\left[x(i)-\mu_{x}\right]^{2}}$.

Skewness: $\frac{\frac{1}{T} \sum_{i=1}^{T}\left[x(i)-\mu_{x}\right]^{3}}{\left(\frac{1}{T} \sum_{i=1}^{T}\left[x(i)-\mu_{x}\right]^{2}\right)^{\frac{3}{2}}}$, asymmetry of the signal probability distribution.

Kurtosis: $\frac{\frac{1}{T} \sum_{i=1}^{T}\left[x(i)-\mu_{x}\right]^{4}}{\left(\frac{1}{T} \sum_{i=1}^{T}\left[x(i)-\mu_{x}\right]^{2}\right)^{3}}-3$, peakedness of the signal probability distribution.

Signal power: $\sum_{i=1}^{T} x(i)^{2}$.

Log-energy: $\sum_{i=1}^{T} \log \left[x(i)^{2}\right]$.

Peak intensity: number of signal peak appearances.

Zero crossings: number of times the signal crosses its median.

Correlation between each pair of axes: $\frac{\sum_{i=1}^{T}\left[x(i)-\mu_{x}\right]\left[v(i)-\mu_{v}\right]}{\sqrt{\sum_{i=1}^{T}\left[x(i)-\mu_{x}\right] \sum_{j=1}^{T}\left[v(j)-\mu_{v}\right]}}$.

Table 2: Statistics used to convert time series windows into feature vectors (taken from [53]). $T$ is the number of ticks in the given time series, $x$ and $v$ are individual axes of the time series data, and $x(i)$ is the $i$ th data tick of $x$. 


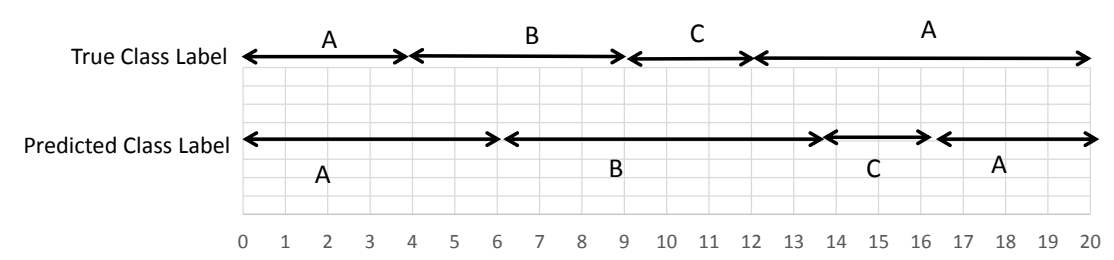

Figure 11: Time series example with 20 ticks from a dataset of three classes A, B and C with true labels and predicted labels.

equations of each statistical features we used in our experiment. We also applied change-point scoring [30] on each axis value and extracted above mentioned 57 statistical features on these. We used efficient feature selection algorithm to find out best features out of $114(57+57)$ features separately.

\subsection{Feature Selection}

Our above mentioned feature extraction method generates 114 statistical features. As we aim to detect infrequent gestural activity as fast as possible, we aim to build our system more computationally efficient excluding the consideration of unnecessary features. Therefore, the goal is to select a minimum number of features that achieve reasonably good classification performance. We use the correlation feature selection (CFS) algorithm to select the subset of features [31]. The CFS algorithm evaluates the worth of a subset of attributes by considering the individual predictive ability of each feature along with the degree of redundancy between them. Subsets of features that are highly correlated with the class while having low intercorrelation are preferred. To identify locally predictive attributes we apply forward Best First Search (BFS). It iteratively adds attributes with the highest correlation in the class as long as there is no other combinations of the attributes which generate a better correlation.

\subsection{Performance Metrics}

To measure the performance of our classification algorithms we used two performance metrics, accuracy and detection time. Accuracy is computed by counting the number of correctly predicted ticks for each true window separately, summing the counts, and dividing by the total number of ticks. From Fig 11, the accuracy of the prediction is given as follows:

$$
\text { Accuracy }=\frac{C P T\left(A_{1}\right)+C P T(B)+C P T(C)+C P T\left(A_{2}\right)}{(\text { Totalnumberofticks })}=\frac{3+3+0+4}{20}=50 \%
$$

where $C P T($.$) is the number of correctly predicted ticks in an interval, and A_{1}$ and $A_{2}$ are the true class "A" windows.

Detection time is computed by counting how many ticks are required for a prediction algorithm to start correctly predicting the class, after a true window begins. From Fig 11 the true window begins at tick 1 (class $A$ ), the algorithm predicts $A$ immediately, so the detection time for that window is 0 . Over the second true window beginning at tick 4 (class $B$ ), the algorithm does not start predicting B until tick 6 so the detection time for that window is $6-4=2$ and so on. Thus the average detection time over the time series segment is:

$$
\frac{(0+2+3+4) \text { ticks }}{4 \text { windows }}=\frac{9}{4} \text { ticks per window }
$$

\subsection{Approaches}

Activity recognition using accelerometer data is a rapidly emerging field with many real-world applications. We apply accelerometer data based activity recognition for infrequent gestural behavior analysis and detection. Though much of the prior works in this area has assumed that the accelerometer data has already been segmented into pure activities, in reality, activity recognition would need to be applied to "free-living" data, which is collected over continuous time period and would consist of a mixture of activities. We applied our proposed novel change-point segmentation based method and compare their performances with traditional fixed segment based HMM method as follows. 


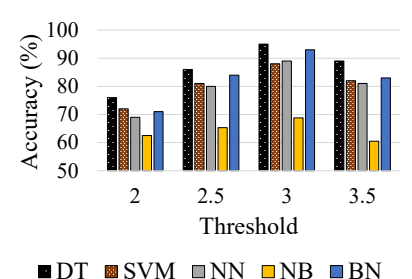

(a)

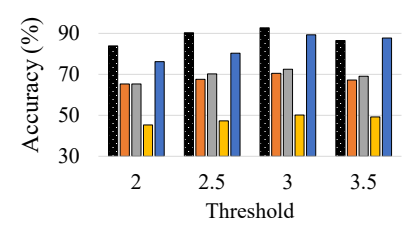

DT $\square \mathrm{SVM} \square \mathrm{NN} \square \mathrm{NB} \square \mathrm{BN}$

(c)

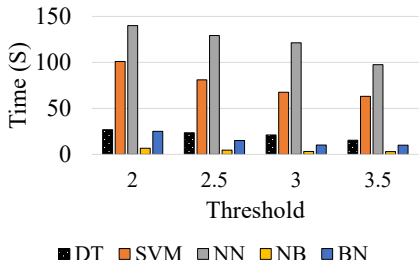

(b)

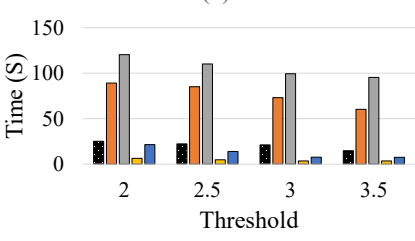

aT $\square \mathrm{SVM} \square \mathrm{NN} \square \mathrm{NB} \square \mathrm{BN}$

(d)

Figure 12: Comparisons of different base classifiers with the increasing of change-point score segmentation threshold with features from raw sensor (a) accuracy vs threshold (b) detection time vs threshold and features from change-point scores of time series data stream (c) accuracy vs threshold (d) detection time vs threshold

\subsubsection{Change-Point Scoring based Segmentation}

In this approach, we used [30] written Matlab implementation of relative density ratio based change-point scoring algorithm [39] (explained in section 5). Once change-point detection scores were generated, we tested a number of threshold values that determined which scores were high enough to be considered a predicted change-point. Threshold values were chosen by considering the false positive rates of change prediction for the change-point detection algorithms. A smaller false positive rate corresponded to a higher and more conservative threshold, which split the time series into fewer segments. A larger false positive rate corresponded to a lower threshold, which split the time series into more segments. After segmenting the data, we extracted features (114 features), applied feature selection and finally classify using mostly explored classifiers for IGAs recognition. Towards the classification, we split data stream into three equal parts: training, validation and testing.

\subsubsection{HMM Based Classification}

In this method, we partitioned each time series into small non-overlapping windows, where each window corresponded to a discrete time index in the HMM (explained in section 6). Then we applied feature extraction on each window. We build and tunes classification models (SVM, C4.5 and Neural Networks) to select observation state. We split data into 4 equal parts: training base classifier, validation, training (HMM), and testing. Here we formulated the problem of making predictions on the testing set in terms of an HMM by treating the second training set as a training HMM. In our dataset we let $X$ be the ground truth activity classes of the windows, and $O$ be the predicted activity classes of the windows, where the predictions were made by the classifiers trained on the first training set. We used the procedure above to calculate $\hat{T}$ and $\hat{S}$, and assumed that these estimates held for the testing set as well as the second training set. We then used the tuned base classifier to predict on the testing set, giving us $O$. Finally, we used $\mathrm{O}, \hat{T}$ and $\hat{S}$ to run the Viterbi algorithm on the testing set and predict the ground truth activity classes $X$.

\subsection{Evaluation}

We tested our approaches with five base classifiers: decision tree, support vector machines, neural networks, naive bayes and bayesian network. We used WEKA API in java platform for our experiments. For decision tree, we chose C4.5 (J48 in WEKA API) with a confidence factor of 0.25. J48 implementation of WEKA API provides auto pruning to tune the classifier which helped avoid the usage of validation dataset. We used [68] implementation of support vector machine called sequential minimal optimization (SMO). For neural network, we used the iteration number of 1000 with 100 hidden layers. We implemented Hidden Markov Model (HMM) in java platform using HMMWeka [17] package. The details results of our two approaches are described as follows. 


\subsubsection{Change-point scoring Segmentation Method}

In this approach, we followed two different strategy. First, we have extracted 57 pre-defined features using the raw sensor data stream ( $x, y$ and $z$ axis values) and used CFS algorithm to choose 13 best features for target classifiers. In Fig 12 (a) and (b), we illustrated details performance metrics (accuracy and detection time) of our chosen 5 different classifiers with increasing of change-point score threshold for segmentation. Second, we have extracted 57 features using the change-point score of the data stream and used CFS algorithm to choose 11 best features for final classification. Fig 12(c) \& (d) presented details performance metrics of our base classifiers with increasing of change-point score. Fig 12 clearly shows that change-point score threshold 3 gives the best performance in each base classifier. We also can say that though Naive Bayes classifier performs better in terms of detection time, Decision Tree algorithm outperforms other classification algorithms as it provides the most accurate classification and close to best detection time.
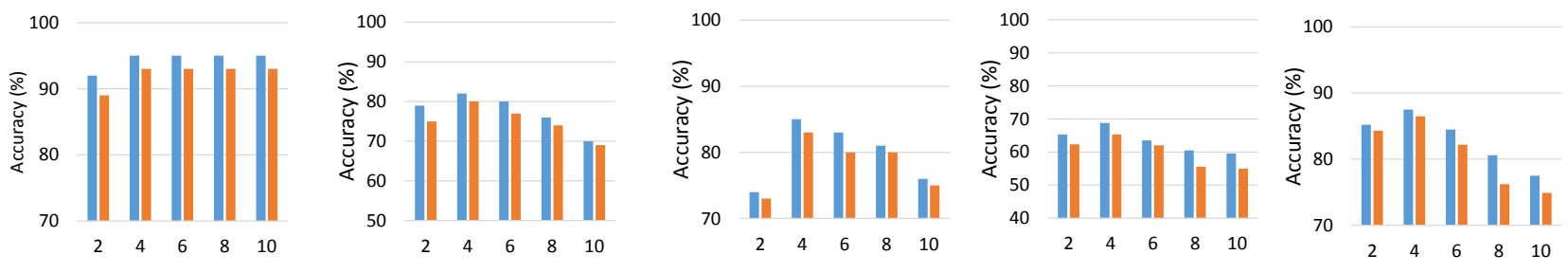

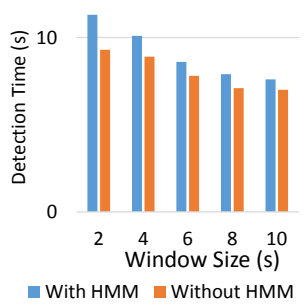

(a)

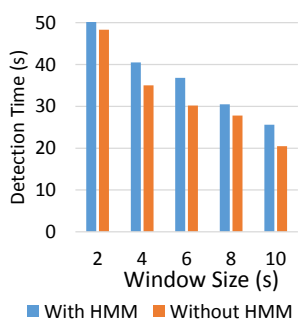

(b)

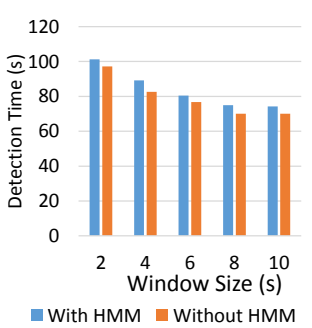

(c)

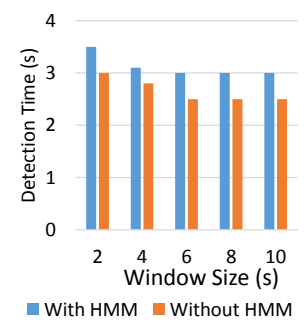

(d)

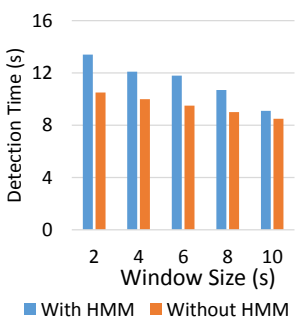

(e)

Figure 13: Comparisons of HMM performances (accuracy on the top and detection time on the bottom) with the increasing of window sizes based on different base classifiers (a) Decision Tree (b) Support Vector Machine (c) Neural Network (d) Naive Bayes (e) Bayesian Networks

\subsubsection{HMM Based Method}

In this approach, we split each time series into windows of fixed length corresponding to discrete time 'ticks' in an HMM, and results for windows of length $2 ; 4 ; 6 ; 8 ; 10$ seconds are shown in Fig 13 . We can see the window size of 4 seconds always gives better classification accuracy as Decision Tree outperforms other base classifiers. It also depicts that in every case, incorporating HMM increases the accuracy measure for all base classifier. Meanwhile, the increment of window size is inversely proportional to the detection time.

\subsubsection{Analysis and Comparison}

From the above results, we can see, for change-point scoring based segmentation method, the best performance can be achieved if we use raw sensor data stream for feature extraction and change-point score threshold of 3 for segmentation with Decision Tree classification algorithm. On the other hand, for HMM based classification method, we can achieve best performance if we use raw sensor data stream and 4 seconds window size of segmentation with Decision Tree classification algorithm as base classifier. For first method, we achieved maximum accuracy of $\approx 95 \%$ with 21.1 seconds of detection time on average. On the other hand, for HMM based method, we achieved maximum accuracy of $\approx 96 \%$ with 10.5 seconds of detection time on an average. Table 3 and 4 show the class level true positive rate (TP rate), false positive rate (FP rate), recall, precision and F-measure from the 10-fold cross validation experiment of our both of the methods (method 1 and method 2 respectively). Though, our first method shows significant accuracy, 
The second approach slightly outperformed in terms of both accuracy and detection time. HMM based approach does not include the computation of a change-point detection score, but does include a final step the computation (via the Viterbi algorithm) of the set of hidden states in an HMM that are most likely to correspond with classifier predictions on the windows in the test data. The Viterbi algorithm is an offline algorithm that considers all of the windows in the time series rather than just the latest window, but it nonetheless runs quickly.

From the above results, it is really difficult to disseminate that HMM based model outperforms change-point detection based model as the outperformance is not significant $(\approx 1 \%)$. To expurgating the comparison more clearly, we reduce our sampling rate from 22 to 2 samples per second and analyze the accuracy and detection time for both of our approaches illustrated in Fig 14. From Fig 14, we can depict that for low sampling rate, change-point score based classification outperforms HMM based approach. Fig 15 shows the ROC curve for both of our method that clearly depicts that change-point based method outperforms HMM based method significantly. However, Fig 15 also depicts that how correlation feature selection can improve the classification accuracies for both change-point and HMM based method. From Table 5 we see that our models outperform other existing solutions in detecting and recognizing

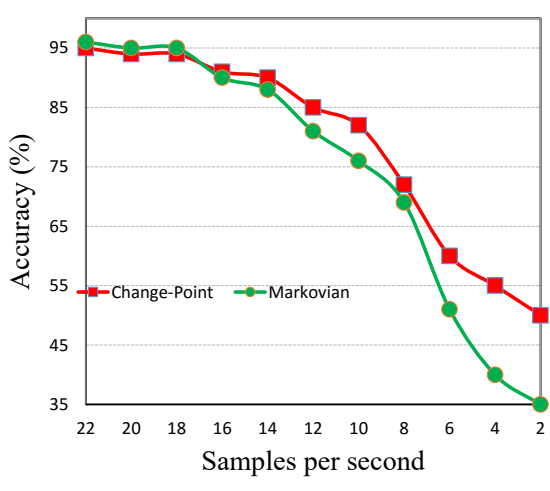

(a)

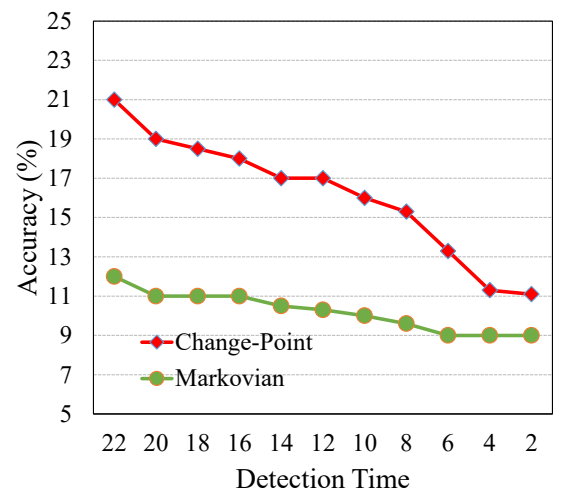

(b)

Figure 14: Comparisons of HMM and change-point based classification performances with the decreasing of sampling rate in terms of (a) accuracy and (b) detection time

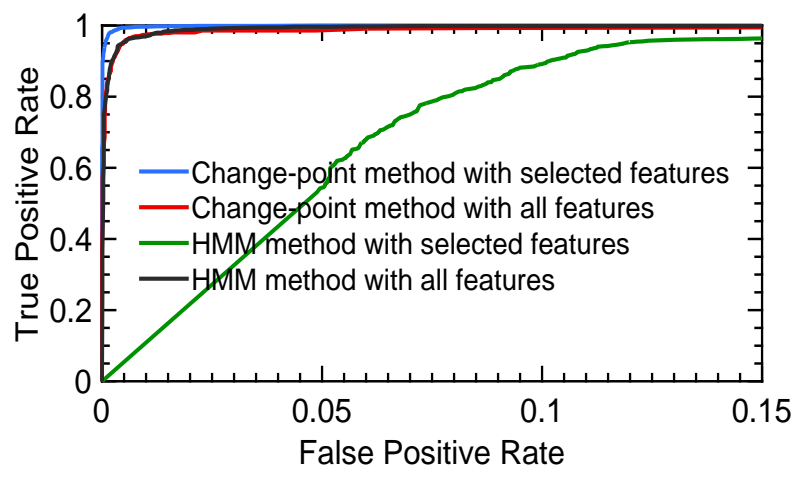

Figure 15: ROC curve true positive vs false positive rate

different types of gestural activities.

\subsubsection{Performance on Benchmark Datasets}

It has become really hard to come up with a firm conclusion that our proposed change-point detection based segmentation outperforms existing HMM based method significantly in lower sampling rate from the above analysis. 
Table 3: The TP rate, FP rate, Precision, Recall and F-measure for each class from the LOPO experiment using C4.5 as classifier and change-point score as frame-level features

\begin{tabular}{|c|c|c|c|c|c|}
\hline Accuracy & TP Rate & FP Rate & Precision & Recall & F-Measure \\
\hline Silence & $95.7 \%$ & $5.8 \%$ & $96.4 \%$ & $95.7 \%$ & $96.0 \%$ \\
\hline Coughing & $86.0 \%$ & $00.3 \%$ & $84.0 \%$ & $86.0 \%$ & $85.0 \%$ \\
\hline Yawning & $90.5 \%$ & $0.1 \%$ & $93.8 \%$ & $90.5 \%$ & $92.1 \%$ \\
\hline Deglutition & $88.5 \%$ & $1.9 \%$ & $85.8 \%$ & $88.5 \%$ & $87.1 \%$ \\
\hline Talking & $96.5 \%$ & $1.2 \%$ & $95.9 \%$ & $96.5 \%$ & $96.2 \%$ \\
\hline Weighted Avg. & $\mathbf{9 5 . 1 \%}$ & $\mathbf{4 . 1 \%}$ & $\mathbf{9 5 . 3 \%}$ & $\mathbf{9 5 . 1 \%}$ & $\mathbf{9 5 . 1 \%}$ \\
\hline
\end{tabular}

Table 4: The TP rate, FP rate, Precision, Recall and F-measure for each class from the HMM Experiment

\begin{tabular}{|c|c|c|c|c|c|}
\hline Accuracy & TP Rate & FP Rate & Precision & Recall & F-Measure \\
\hline Silence & $97.8 \%$ & $7.5 \%$ & $96.4 \%$ & $97.5 \%$ & $97.1 \%$ \\
\hline Coughing & $88.4 \%$ & $00.7 \%$ & $90.1 \%$ & $88.4 \%$ & $89.2 \%$ \\
\hline Yawning & $81.5 \%$ & $0.3 \%$ & $89.5 \%$ & $87.3 \%$ & $88.4 \%$ \\
\hline Deglutition & $87.3 \%$ & $0.5 \%$ & $89.5 \%$ & $87.3 \%$ & $85.8 \%$ \\
\hline Talking & $92.4 \%$ & $1.3 \%$ & $93.0 \%$ & $92.4 \%$ & $92.7 \%$ \\
\hline Weighted Avg. & $\mathbf{9 5 . 8 \%}$ & $\mathbf{5 . 7 \%}$ & $\mathbf{9 6 . 1 \%}$ & $\mathbf{9 6 . 3 \%}$ & $\mathbf{9 6 . 0 \%}$ \\
\hline
\end{tabular}

To strengthen our method's outperformance precisely, we choose two benchmark datasets with high sampling rate, HHAR [56] and REALDISP [57], to evaluate our novel change-point detection based segmentation method in terms of sampling rate.

HHAR dataset consists of 6 postural activities (biking, sitting, standing, walking, stair up and stair down), accelerometer and gyroscope sensors integrated 12 wearable devices ( 4 smartwatches, 8 smartphones) with 9 users. We choose only one Samsung Galaxy S3 smartphone integrated accelerometer sensor streams (sampling frequency 100 $\mathrm{Hz}$ ) placed in the right pocket of each user for detecting 6 postural activities performances to evaluate the efficiency of our approach. On the other hand, REALDISP datasets consists of 33 activities, multi sensors (accelerometer, gyroscope and magnetometer) integrated wearable devices in 9 difference nodes of the body with 17 users. We choose right thigh worn wearable sensor device integrated accelerometer signal streams (sampling frequency $50 \mathrm{~Hz}$ ) and 6 activities (walking, jogging, running, jump up, jump sideways, and cycling) dataset to evaluate our approach's efficiency.

For change-point detection based approach, we apply change-point scores and find the segments, apply accelerometer feature extraction and feature selection to extract 12 best features then finally apply decision tree algorithm to evaluate results. For fixed length segmentation technique, we first set the segment window to 4 seconds, apply accelerometer feature extraction and feature selection to extract 12 best features and apply HMM method (explained before) for classifying activities.

In REALDISP dataset, classification accuracies with change-point based decision tree method shows $86.9 \%$ (FP rate $11.5 \%$ ) while fixed length segmentation based HMM algorithm shows $82.3 \%$ (FP rate $13.3 \%$ ). On the other hand,

Table 5: Comparison with prior works' classification recall measure

\begin{tabular}{|c|c|c|c|c|}
\hline Methods & Hayley Hung [16] 2013 & BodyBeat [25] 2014 & $1^{\text {st }}$ approach & $2^{\text {nd }}$ approach \\
\hline Silence & N/A & $74.38 \%$ & $95.9 \%$ & $97.6 \%$ \\
\hline Coughing & N/A & $80.0 \%$ & $82.7 \%$ & 88.4 \\
\hline Yawning & $24 \%$ & $75.0 \%$ & $80.2 \%$ & $81.5 \%$ \\
\hline Deglutition & $21 \%$ & $72.09 \%$ & $81.1 \%$ & $87.3 \%$ \\
\hline Talking & $82.0 \%$ & $81.06 \%$ & $89.0 \%$ & $92.4 \%$ \\
\hline Weighted Avg. & N/A & $\mathbf{7 1 . 2 \%}$ & $\mathbf{9 5 . 3 \%}$ & $\mathbf{9 6 . 7 \%}$ \\
\hline
\end{tabular}



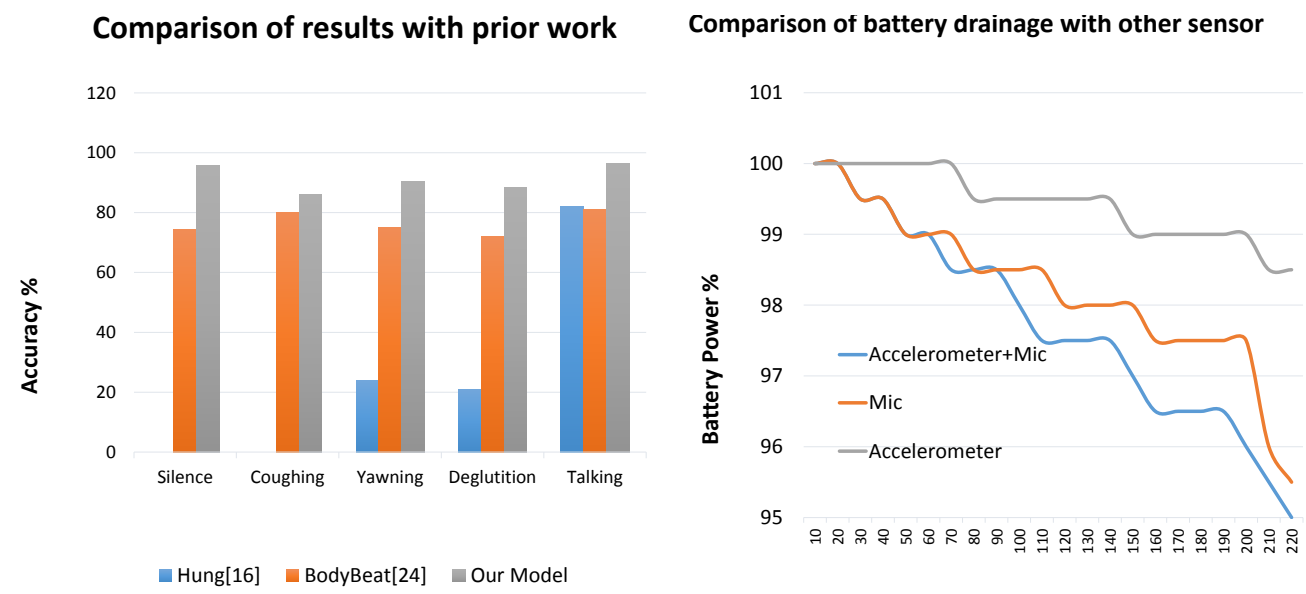

Figure 16: (a) Comparison of Results with prior works (b) Comparison of battery power drop of sensors

on HHAR dataset, our change-point segmentation based decision tree algorithm shows 93.4\% (FP rate 3.5\%) accuracy while HMM based classification accuracy stagnates at $87.3 \%$ (FP rate 9.3\%). We downsample the sensor signal by a fixed factor to test the algorithms performances in lower sampling frequency. Fig 17 shows the comparisons of the accuracies for both of our method and HMM method on two benchmark datasets which clearly depicts that in lower sampling rate, our method outperforms traditional fixed length based HMM method significantly.

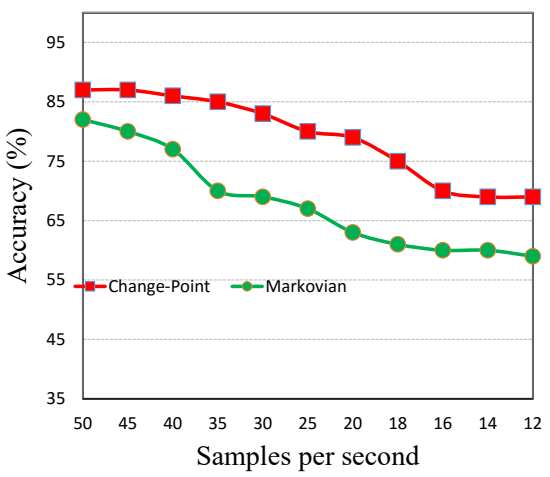

(a)

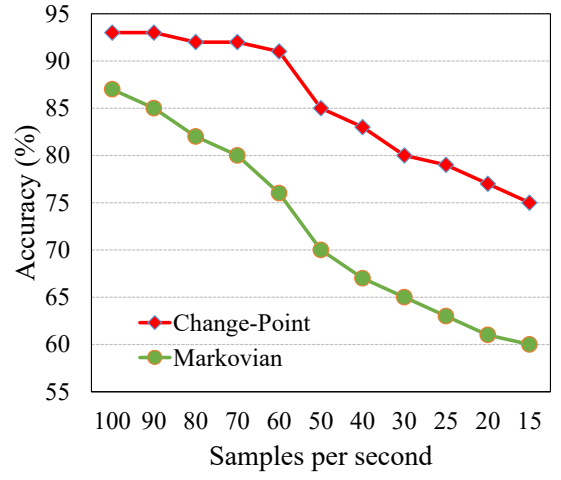

(b)

Figure 17: Comparisons of change-point segmentation method based decision tree algorithm and fixed length segmentation based HMM algorithm accuracies on (a)REALDISP and (b) HHAR datasets

\section{Discussion}

The correlation between the body motion and social behavior of the people has been well-established by the social psychologists [26, 27, 28]. Existing research in social psychology also highlights a strong correlation between the speech and body gestures among the speaker and listener $[27,28]$. In lower sampling rate, the traditional accelerometer feature based activity recognition fails to achieve higher accuracy for infrquent gesture recognition because each infrequent gestural activity executes completely different pattern with different episode duration. Fixed length segmentation fails to find fine-grained classification accuracy which necessitates to apply different segmentation for different activity classification. In this work, we propose two approaches for gestural activity recognition using only a single energy efficient sensor, accelerometer embedded in an ubiquitous earring. We show that CPS approach outperforms the 
fixed length based traditional activity recognition methods in terms of accuracy and close enough in terms of detection time. GeSmart also attests significant energy savings and higher detection accuracy compared to the existing methods (Fig. 16). We do more microscopic analysis of 'coughing' activity recognition performance to evaluate our proposed change-point segmentation based method and fixed length segmentation based HMM method. If we consider, three 'coughing' states (single, two and three consecutive couching) as three classes, then our total classification problem consists of 7 classes ('silence', 'talking', 'single coughing', 'consecutive two coughing', 'consecutive three coughing', 'yawning' and 'deglution'). Fig 18 shows the classification accuracies for 7 class problem of the two approaches which clearly depicts that detection accuracy of 'consecutive three coughing' is much higher for both of the method followed by 'consecutive two coughing' and 'single coughing' IGAs. However, it also can be seen that our method outperforms for both overall and individual coughing IGAs classification accuracies. To test our feature selection performance, we train

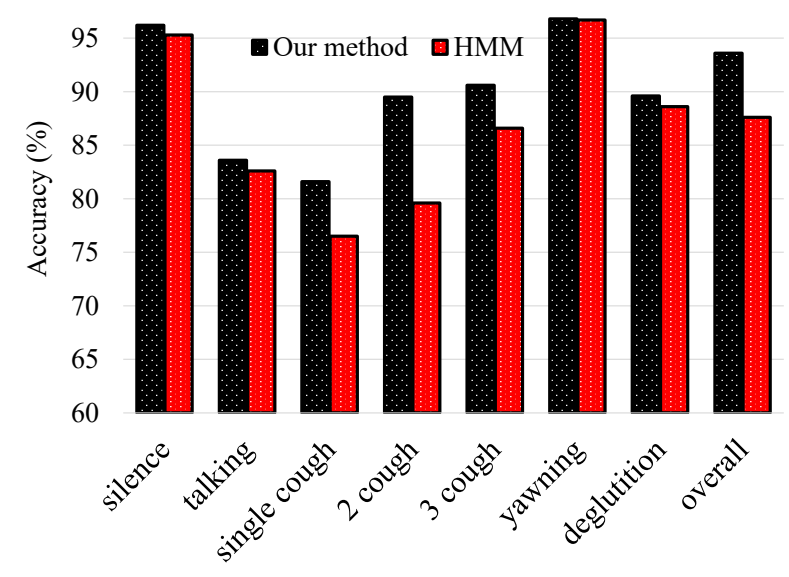

Figure 18: Accuracy comparison for 7-class problem based on our proposed method and fixed length HMM method

Privacy and energy efficiency: The larger group deployment reinforced the importance of considering privacy aspects of data logging, collection and analysis. Collecting sensor data, particularly from microphone or camera, involves recording people in unconstrained and unpredictable situations, both in public and private space. It may include the recording of unnecessary audio or video information without proper consent of the users which is unethical and often illegal. Hence, most of the people are reluctant of wearing some devices which capture audio or video of ADLs. Our system conforms user's privacy by avoiding audio or video recording. Meanwhile, only using accelerometer can reduce significant amount of energy drainage which is always a bottleneck. Fig. 16 (b) shows a simple measure of battery power drainage of different sensors in Google Nexus 4 smartphone. It shows that accelerometer sensor help improve the battery life of smartphone 2.3 and 3.3 times respectively compared to an audio and audio cum accelerometer sensor based activity recognition approach.

\section{Conclusion and Future Work}

Older adults' health safety assurance has become increasingly important as the number of older adults living worldwide and average life expectancy of them increases. In this paper, we have exploited the significance of IGAs on older adults health-care and presented GeSmart, an energy efficient infrequent GAR model to predict the chronic behavioral conditions. We advocate that microscopic gestural activity recognition can provide useful insights for long-term care and behavioral health. We explored one online (CPS) and one offline (HMM) method towards hybrid classification approaches based on change-point detection method and compared their performance according to our pre-defined performance metrics. Change-point detection is a field of statistics popular in control theory and other similar applications. This method takes as input an initial time series and partitions it into smaller pieces using change-point detection. While, regular activity recognition can be exploited easily using fixed length window based 
traditional activity recognition algorithm, infrequent gestural level activity recognition fails with it in terms of lower sampling rate which is usual case for available cheap devices. We are the first of a kind used Change-point scoring based segmentation to provide more fine-grained classification accuracy for IGAs detection than existing works. The best performed method outperforms the previous IGA detection method's accuracy by over $24 \%$ [25]. We have also shown through extensive experimentations with a variety of IGAs that the position ear is a viable option to consider for capturing slightest perturbation of gestural signal in presence of regular ADLs.

The objective of this work is to make an energy efficient, computationally fast and accurate classification of activities which are subjected to be abrupt changes (infrequent non-speech gestures like coughing, deglution and yawning) and distinguish them from other frequent gestures (such as talking and silence). For any sensor device, sensing with low sampling rate is one of the primary way of reducing computational cost and energy consumption. However, low sampling rate costs the accuracy which can be reduced using proper segmentation rather than using fixed length segmentation. We propose a new segmentation technique (change-point detection based) and compare its performance with the most popular fixed length segmentation based technique (HMM). We plan to explore the possible less energy consuming classifiers (such as Dynamic Bayesian Network) for designing energy-efficient smart devices in the form of jewelries. We also plan to test our model on real target age group such as older adults in an uncontrolled environment. Finally, based on the early potential results reported in this work, we plan to bring GeSmart to life by using it for several healthcare applications such as agitation detection for Alzheimer's patients or tremor detection for Parkinson's patients. The ability to recognize IGAs using just the motion sensor opens up the potential for recognizing and analyzing people's activities of daily living (ADL) without explicitly capturing other costly sensor data and paying for computational overhead. For minimizing the ground truth collection and large scale deployment, we plan to apply active learning and transfer learning techniques along with our proposed approaches.

Our device is customized in the lab and not commercially marketable. The device needs more miniaturization and light weight conversion to use it as an ubiquitous wearable earring for older adults. Our initial goal of this study is to find out the opportunity to detect IGAs using only a single accelerometer integrated earring with very lower sampling rate thus the data length and transmission consume less power. That is why, we customize a device that facilitates a controllable sampling rate. In our future goal, we will build a complete, light weight, energy efficient, micro accelerometer sensor integrated earring that can come up with all commercial requirements.

\section{Acknowledgment}

This work is supported partially by the NSF Award \#1344990, UMB-UMBC Research and Innovation Partnership Grant, and Constellation $E^{2}$ : Energy to Educate Grant.

\section{References}

[1] Nirmalya Roy, Archan Misra, Diane Cook, Infrastructure-assisted smartphone-based ADL recognition in multi-inhabitant smart environments, PerCom, 2013, $38-46$

[2] JH Bergmann, AH McGregor, Body-worn sensor design: What do patients and clinicians want?, Annals of Biomedical Engineering, 2011, 39, 9, 2299-2312

[3] S. M. Shahriar Nirjon, Robert F. Dickerson, Qiang Li, Philip Asare, John A. Stankovic, Dezhi Hong, Ben Zhang, Xiaofan Jiang, Guobin Shen, Feng Zhao, MusicalHeart: a hearty way of listening to music, SenSys, 2012, 43-56

[4] S. S. Intille, Using a live-in laboratory for ubiquitous computing research, PERVASIVE, 2006, LNCS , 3968

[5] N. Gyorbiro, A. Fabian, G. Homanyi, An activity recognition system for mobile phones, Mobile Networks and Applications, 2008, 14, 82-91

[6] J. Kwapisz, G. Weiss, S. Moore, Activity recognition using cell phone accelerometerse, International Workshop on Knowledge Discovery from Sensor Data, 2010, $10-18$

[7] Suman Nath, ACE: exploiting correlation for energy-efficient and continuous context sensing, MobiSys, 2012, 28, 29-42

[8] M. Coulson, Attributing emotion to static body postures: recognition accuracy, confusions, and viewpoint dependence, Journal of Nonverbal Behavior, 1992, 28, $2,117-139$

[9] H. Gunes, M. Piccardi, Bi-modal emotion recognition from expressive face and body gestures, Journal of Network and Computer Applications, 2007, 30, 4, $1334-1345$

[10] Ginevra Castellano, Loic Kessous, George Caridakis, Multimodal emotion recognition from expressive faces, body gestures and speech, Artificial Intelligence and Innovations 2007: from Theory to Applications IFIP The International Federation for Information Processing , 2007, 247, 375-388 
[11] R. Cowie, E Douglas-Cowie, Automatic statistical analysis of the signal and prosodic signs of emotion in speech, Fourth International Conference on Spoken Language, 1996. ICSLP 96. Proceedings, 1996, 3, 1989-1992

[12] Xia Mao, Bing Zhang, Yi Luo, Multi-level Speech Emotion Recognition Based on HMM and ANN, WRI World Congress on Computer Science and Information Engineering, 2009, 7, 225-229

[13] Xin Min Cheng, Pei Ying Cheng,Li Zhao, A Study on Emotional Feature Analysis and Recognition in Speech Signal, Proceedings of the International Conference on Measuring Technology and Mechatronics Automation (ICMTMA), 2009, 1, 418-420

[14] K. H. Kim, S. W. Bang, S. R. Kim, Emotion recognition system using short-term monitoring of physiological signals, Medical and Biological Engineering and Computing , 2004, 42, 419-427

[15] R. W. Picard, E. Vyzas , J. Healey, Toward machine emotional intelligence: analysis of affective physiological state, IEEE Transactions on Pattern Analysis and Machine Intelligence, 2001, 23, 1175-1191

[16] Hayley Hung, Gwenn Englebienn, Jeroen Kools, Classifying social actions with a single accelerometer, UbiComp '13 Proceedings of the 2013 ACM international joint conference on Pervasive and ubiquitous computing, 2013, 207-210

[17] HMMWeka, Marco Gillies, http://doc.gold.ac.uk/ mas02mg/software/hmmweka/

[18] I.A. Essa, A.P. Pentland, Coding, analysis, interpretation, and recognition of facial expressions, IEEE Transactions on Pattern Analysis and Machine Intelligence, $1997,19,757-763$

[19] Ying-Li Tian, T. Kanade, J.F. Cohn, Recognizing action units for facial expression analysis, IEEE Transactions on Pattern Analysis and Machine Intelligence , 2001, 23, 97-115

[20] T. Choudhury, A. Pentland, Characterizing Social Networks using the Sociometer, NAACOS, 2004

[21] C.-C. Lian and J. Y.-j. Hsu, Probabilistic Models for Concurrent Chatting Activity Recognition, IJCAI, 2009, 1138-1143

[22] F. S. Wei, L. C. D. Silva,, Speech based emotion classification, International Conference on Electrical Electronic Technology,, 2001, 1, 297-301

[23] Oh-Wook Kwon, Kwokleung Chan, Jiucang Hao, Te-Won Lee, Emotion Recognition by Speech Signals, In proceeding of: 8th European Conference on Speech Communication and Technology, EUROSPEECH, 2003, 125-128

[24] L.C De Silva, Pei Chi Ng, Bimodal emotion recognition, Fourth IEEE International Conference on Automatic Face and Gesture Recognition, 2000. Proceedings. , $2000,332-335$

[25] T. Rahman, A. Adams, E. Carroll, B. Zhou, H. Peng, Mi Zhang, T. Choudhury, BodyBeat: A Mobile System for Sensing Non-Speech Body Sounds, International Conference on Mobile Systems, Applications and Services (MobiSys), 2014

[26] T. L. Chartrand and J. A. Bargh, The chameleon effect: the perception-behavior link and social interaction, Journal of Personality and Social Psychology, 1999, $76,6,893-910$

[27] A. Kendon, Conducting Interaction: Patterns of Behavior in Focused Encounters, Cambridge University Press, 1990

[28] D. McNeill, Language and Gesture, Cambridge University Press New York, 2000

[29] McGarvey LP, Patterns of cough in the clinic,Pulm Pharmacol Ther. 2011 Jun;24(3):300-3

[30] Song Liu, Makoto Yamada, Nigel Collier, Masashi Sugiyama, Change-point detection in time-series data by relative density-ratio estimation, Neural Networks. $2013 ; 72-83$

[31] Hall, M. A. Correlation-based Feature Selection for Machine Learning. PhD Thesis (April 1999).

[32] Kohavi, Ron. A study of cross-validation and bootstrap for accuracy estimation and model selection. Proceedings of the Fourteenth International Joint Conference on Artificial Intelligence, 1995 (San Mateo, CA: Morgan Kaufmann) 2 (12): 11371143.

[33] Fehmi Ben Abdesslem, Andrew Phillips, and Tristan Henderson.Less is more: energy-efficient mobile sensing with senseless. MobiHeld, page 61-62. ACM, (2009)

[34] Y. Kawahara, T. Yairi, and K. Machida. Change-point detection in time-series data based on subspace identification. In Proceedings of the 7th IEEE International Conference on Data Mining, pages 559564, 2007

[35] S. M. Ali and S. D. Silvey. A general class of coefficients of divergence of one distribution from another. Journal of the Royal Statistical Society, Series B, 28(1):131142, 1966.

[36] K. Pearson. On the criterion that a given system of deviations from the probable in the case of a correlated system of variables is such that it can be reasonably supposed to have arisen from random sampling. Philosophical Magazine, 50:157175, 1900.

[37] M. Yamada, T. Suzuki, T. Kanamori, H. Hachiya, and M. Sugiyama. Relative densityratio estimation for robust distribution comparison. Neural Computation, 2013. to appear.

[38] Chronos: http://processors.wiki.ti.com/index.php/EZ430-Chronos

[39] source code: http://www.makotoyamada-ml.com/RuLSIF.html

[40] bosch: http://www.bosch-sensortec.com/de/homepage/products_3/3_axis_sensors/acceleration_sensors/bma250_1/bma250 
[41] Li, Z., Exercises Intensity Estimation Based on the Physical Activities Healthcare System, bookCommunications and Mobile Computing, 2009. CMC'09. WRI International Conference on, volume3, pages132-136, year2009, organizationIEEE

[42] James. R. Anderson, Pauline Meno, Psychological Influences on Yawning in Children, Current psychology letters, 2003.

[43] Rothney, M.P. and Neumann, M. and Béziat, A. and Chen, K.Y., An artificial neural network model of energy expenditure using nonintegrated acceleration signals, journalJournal of Applied Physiology, volume103, number4, pages1419-1427, year2007, publisherAm Physiological Soc

[44] Patrick S. Hamilton and E.P. Limited. Open Source ECG Analysis Software Documentation. 2002.

[45] J. Fogarty, C. Au, and S.E. Hudson. Sensing from the basement: a feasibility study of unobtrusive and low-cost home activity recognition. In Proceedings of the 19th annual ACM symposium on User interface software and technology, UIST '06, pages 91-100, New York, NY, USA, 2006. ACM.

[46] Andreas Krause, Matthias Ihmig, and et al. Trading off prediction accuracy and power consumption for context-aware wearable computing, ISWC 2005.

[47] David Chu, Nicholas D.Lane, and et al. Balancing energy, latency and accuracy for mobile sensor data classification. In Proc. of Sensys 2011.

[48] J. Rowan and E.D. Mynatt. Digital family portrait eld trial: Support for aging in place. In Proceedings of the SIGCHI Conference on Human Factors in Computing Systems, CHI '05, pages 521-530, New York, NY, USA, 2005. ACM.

[49] T.V. Duong, H.H. Bui, D.Q. Phung, and S. Venkatesh. Activity recognition and abnormality detection with the switching hidden semi-markov model. In IEEE Computer Society Conference on Computer Vision and Pattern Recognition, pages 838-845, June 2005.

[50] L. Bao and S.S. Intille. Activity recognition from user-annotated acceleration data. pages 1-17. Springer, 2004.

[51] Staudenmeyer, J. and Pober, D. and Crouter, S. and Bassett, D. and Freedson, P., An Artificial Neural Network to Estimate Physical Activity Energy Expenditure and Identify Physical Activity Type from an Accelerometer, Journal of Applied Physiology, 2009, 1300-1307

[52] S. Russell and P. Norvig. Arti cial Intelligence: A Modern Approach, pages 566-583. Prentice Hall, third edition, 2010.

[53] Zheng, Y. and Wong, W.K. and Guan, X. and Trost, S., Physical Activity Recognition from Accelerometer Data Using a Multi-Scale Ensemble Method, book Twenty-Fifth Annual Conference on Innovative Applications of Artificial Intelligence, 2013, publisher IAAI

[54] Michael Mason, Physical activity recognition of free-living data using change-point detection algorithms and hidden Markov models, Masters Thesis, Oregon State University, 2013.

[55] T. Gu, Z. Wu, X. Tao, H.K. Pung, and J. Lu. Epsicar: An emerging patterns based approach to sequential, interleaved and concurrent activity recognition. In Pervasive Computing and Communications, IEEE International Conference on, , 2009

[56] Allan Stisen, Henrik Blunck, Sourav Bhattacharya, Thor Siiger Prentow, Mikkel Baun Kjrgaard, Anind Dey, Tobias Sonne, and Mads Moller Jensen, Smart Devices are Different: Assessing and Mitigating Mobile Sensing Heterogeneities for Activity Recognition, SenSys 2015.

[57] O. Banos, M. A. Toth, M. Damas, H. Pomares, I. Rojas,Dealing with the effects of sensor displacement in wearable activity recognition. Sensors vol. 14, no. 6, 2014.

[58] J.R. Kwapitz, G.M. Weiss, and S. Moore. Activity recognition using cell phone accelerometers. SIGKDD, 12(2):74-82, 2010

[59] K. Grauman. Efficient activity detection with max-subgraph search. In Proceedings of the 2012 IEEE Conference on Computer Vision and Pattern Recognition (CVPR), CVPR '12, IEEE Computer Society, 2012.

[60] S.J. Bae, B.M. Mun, and K.Y. Kim. Change-point detection in failure intensity: A case study with repairable artillery systems. Computers and Industrial Engineering, 64:11-18, January 2013

[61] A.G. Tartakovsky, B.L. Rozovskii, R.B. Blazek, and H. Kim. A novel approach to detection of intrusions in computer networks via adaptive sequential and batchsequential change-point detection methods. IEEE Transactions on Signal Processing, 54:3372-3382, September 2006.

[62] M. Staudacher, S. Telserb, A. Amannc, H. Hinterhuberb, and M. Ritsch-Marte. A new method for change-point detection developed for on-line analysis of the heart beat variability during sleep. Statistical Mechanics and its Applications, 349:582- 596, April 2005

[63] L.E. Baum, T. Petrie, G. Soules, and N. Weiss. A Maximization Technique Occurring in the Statistical Analysis of Probabilistic Functions of Markov Chains. The Annals of Mathematical Statistics, 41(1):164-171, 1970.

[64] Ni-Chun Wang and Hudson, R.E. and Lee Ngee Tan and Taylor, C.E. and Alwan, A. and Rung Yao, Change point detection methodology used for segmenting bird songs,Signal and Information Processing (ChinaSIP), 2013 IEEE China Summit International Conference on, 2013.

[65] Zhang, Chi and Hansen, John HL, Effective segmentation based on vocal effort change point detection, Speech Analysis and Processing for Knowledge Discovery, 2008

[66] X. Nguyen, M. J. Wainwright, and M. I. Jordan. Estimating divergence functionals and the likelihood ratio by convex risk minimization. IEEE Transactions on Information Theory, 56(11):58475861, 2010

[67] M. Sugiyama, T. Suzuki, and T. Kanamori. Density Ratio Estimation in Machine Learning. Cambridge University Press, Cambridge, UK, 2012.

[68] J. Platt: Fast Training of Support Vector Machines using Sequential Minimal Optimization. In B. Schoelkopf and C. Burges and A. Smola, editors, Advances in Kernel Methods - Support Vector Learning, 1998.

[69] Alaiad, A. Zhou, L. Patient Behavioural Intention toward Adopting Healthcare Robots. The 19th Americas Conference on Information Systems (AMCIS), Chicago, USA, 2013.

[70] Alaiad, A., \& Zhou, L. (2015, January). Patients' Behavioral Intentions toward Using WSN Based Smart Home Healthcare Systems: An Empirical Investigation. In System Sciences (HICSS), 2015 48th Hawaii International Conference on (pp. 824-833). IEEE. doi: 10.1109/HICSS.2015.104 